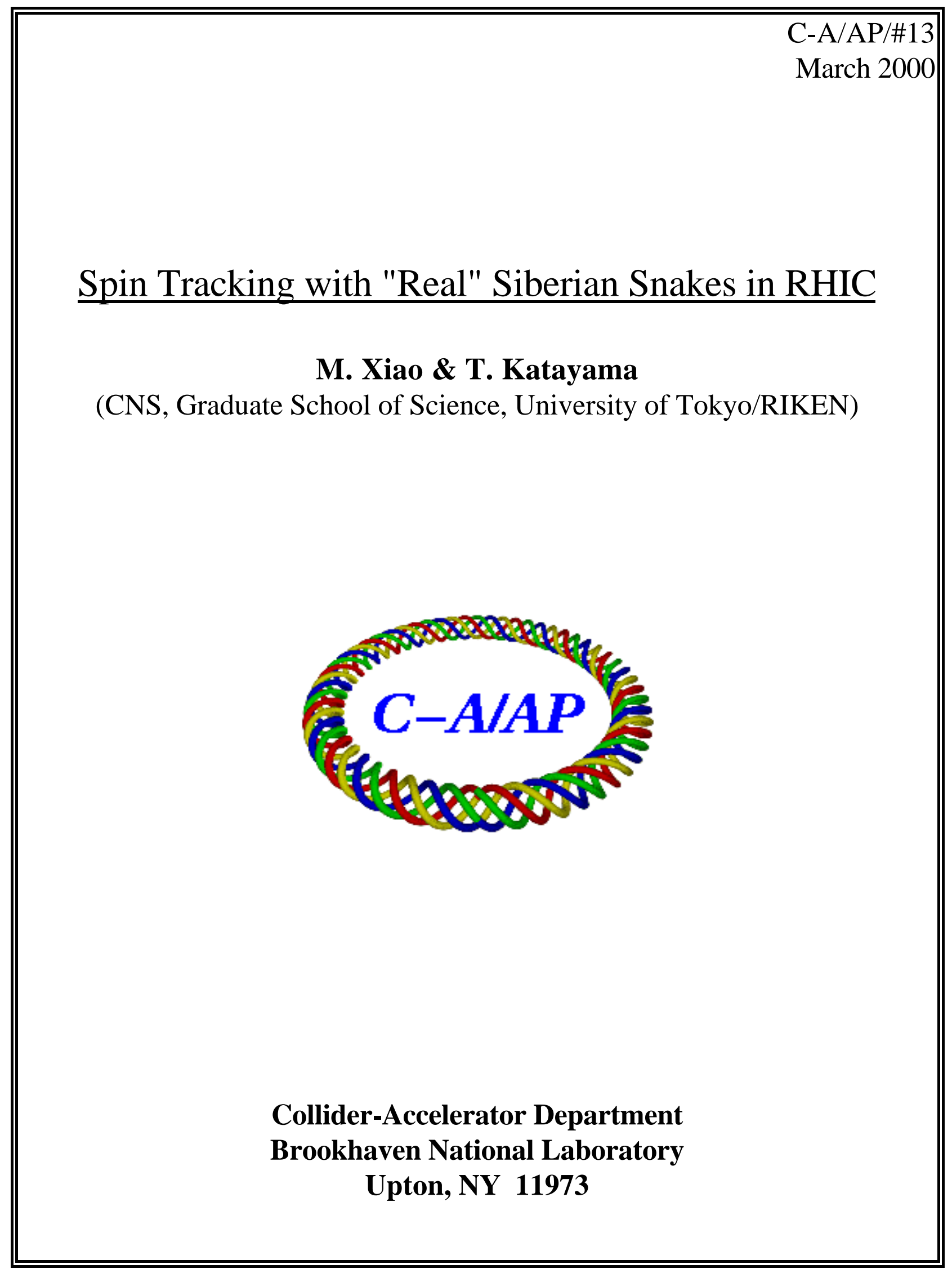




\title{
Spin Tracking with "Real" Siberian Snakes in RHIC
}

\author{
M. Xiao and T. Katayama \\ (CNS, Graduate School of Science, University of Tokyo/RIKEN)
}

Abstract: Spin tracking with "real" Siberian Snakes in RHIC was done. The tracking code SPINK was upgraded in the aspects as follows: (1) the normalized canonical variables $\left(x, p_{x} / p_{s}, y, p_{y} / p_{s},-c \Delta t, p_{t}\right)$ were used, which are consistent to code MAD. (2) Symplectic problem was solved for long term spin tracking. (3) the orbital map for the acceleration of a RF-cavity was obtain to guarantee the invariants through the acceleration to the top energy. (4) special attention was paid to the interpolation for the energy-dependent symplectified numerical orbital maps and spin matrices of Siberian Snakes during the tracking with acceleration. The spin tracking results in the presence of intrinsic depolarization and imperfect depolarization were presented in this report.

\section{Introduction}

The depolarization due to resonances can be studied numerically by tracking the orbital and spin motion of protons in the ring. The spin tracking with Siberian Snakes in RHIC has been carried out by several authors [1, 2], and the intrinsic and imperfection resonances have been found. The general idea of these works was to track a certain number of protons through the machine lattice. Matrices are used to transform orbit and spin coordinates. The orbit matrices were built from a Twiss file, output of the code MAD. However, the tracking was done based on analytical modeling of the magnetic fields for the helical magnets, such as Belewett and Chasman[3]. In addition, since only corrected vertical closed orbit distortions can be read from the Twiss file, the misalignments of the magnets in the lattice and field errors were not taken into account. Furthermore, since the orbital matrices were built from Twiss functions, the coupling effects introduced by helical magnetic field of Siberian Snakes can not be taken into calculations.

Since the strength of spin resonances depends on the distance of a particle from the equilibrium orbit, the depolarization is affected by the closed orbit after the correction by the correctors used in the lattice. This is the indirect effect of the correctors on spin motion. Actually, the correctors have also direct effect on spin motion. That's why the best orbit correction not necessary provides the best spin resonance correction. 
To study these problems, MAD was modified to transmit to the code SPINK the information of the errors of each machine element as well as corrected closed orbit distortion. Code SPINK was improved [4] to accordingly displaces and rotates the corresponding orbit transport maps, and correctors are taken as kick magnets on spin motion. The "transport" orbital map of each element in lattice was directly output from MAD. However, we found the following problems have to be solved when the spin tracking through the acceleration to the top energy is carried out by this version of code SPINK.

(1) The variables used were $\left(x, x, y, y^{\prime}, l, \delta\right)$, which were not canonical variables and not consistent to those used in code MAD, although it took the "Transport" maps of the elements in the lattice directly from the output of MAD.

(2) The emittances are artificially shrinked since the symplectic problem was not taken into account.

(3) The effect of RF-cavity on orbital motion through the acceleration was not put into orbit tracking.

(4) The conventions for magnetic field are not consistent to those in code MAD.

Therefore, the upgrading of code SPINK will be described firstly in this report.

The study of spin precession and orbital motion in a "real" Siberian Snake based on numerically calculated magnetic field by TOSCA [5,6] shows that the spin matrices and orbital maps of a Siberian Snake are energy dependent. The work gave the energydependent numerical orbital matrices and unitary spin matrices of the Siberian Snakes, and the orbital maps of a snake were symplectified. The results are used in spin tracking for the whole acceleration and storage period. The description of the insertion module of field-map generated matrices of "real" Siberian Snakes into RHIC lattice and the trick of the interpolation of energy-dependent symplectified numerical orbital maps and spin matrices during the acceleration are introduced next. Special attention was paid on keeping the symplecticity of the interpolated orbital maps and the unitarity of the interpolated spin rotation maps. Fig. 1 gives the logical diagram of the upgraded Code SPINK. 
In fact, we found that the symplecticity violation of one turn map is $5.6 \times 10^{-6}$ in $x$ direction, and $4.46 \times 10^{-9}$ in $y$-direction. The one turn map is obtained by concatenating the matrices of all the elements in the lattice, except Siberian Snake, from the output of code MAD. Obviously, it was not accurate enough to do the orbital tracking for about $10^{7}$ turns. Therefore, we also solved this problem before doing the spin tracking.

The spin tracking with single- and multi- particles through the acceleration in RHIC with "real" Siberian Snakes, in the presence of both intrinsic depolarization and imperfect depolarization, has be carried out, and the results and discussion will be presented in this report.

\section{Upgraded Code of SPINK}

\subsection{Normalized canonical variables}

SPINK takes the Transport orbital maps from MAD, therefore, the following normalized canonical variables consistent with MAD are used[7],

$$
\left(x, \quad p_{x}=\frac{P_{x}}{p_{s}}\right), \quad\left(y, \quad p_{y}=\frac{P_{y}}{p_{s}}\right), \quad\left(-c \Delta t, \quad p_{t}=\frac{\Delta E}{p_{s} c}\right) .
$$

where $P_{x}$ and $P_{y}$ are transverse momenta, $P_{s}$ is the momentum of a particle, $\Delta t$ is the time relative to the synchronous particle, and

$$
\begin{aligned}
& p_{t}=\frac{\Delta E}{P_{s} c}=\frac{E-E_{o}}{P_{s} c} \\
& P_{s}=P_{o}(1+\delta)
\end{aligned}
$$

here, $E$ is the total energy, $E_{o}$ is the particle energy related to $P_{o}$ (designed energy). $-\mathrm{c} \Delta t$ is canonically conjugate to $p_{t}$.

The relation between the slopes $x^{\prime}, y^{\prime}$ and the normalized momenta $p_{x}, p_{y}$ is

$$
\begin{aligned}
& x^{\prime} \approx p_{x}\left(1+h x-p_{t} \boldsymbol{\beta}\right), \\
& y^{\prime} \approx p_{y}\left(1+h x-p_{t} \boldsymbol{\beta}\right)
\end{aligned}
$$

where $h$ is the curvature of the reference orbit in the mid-plane. 


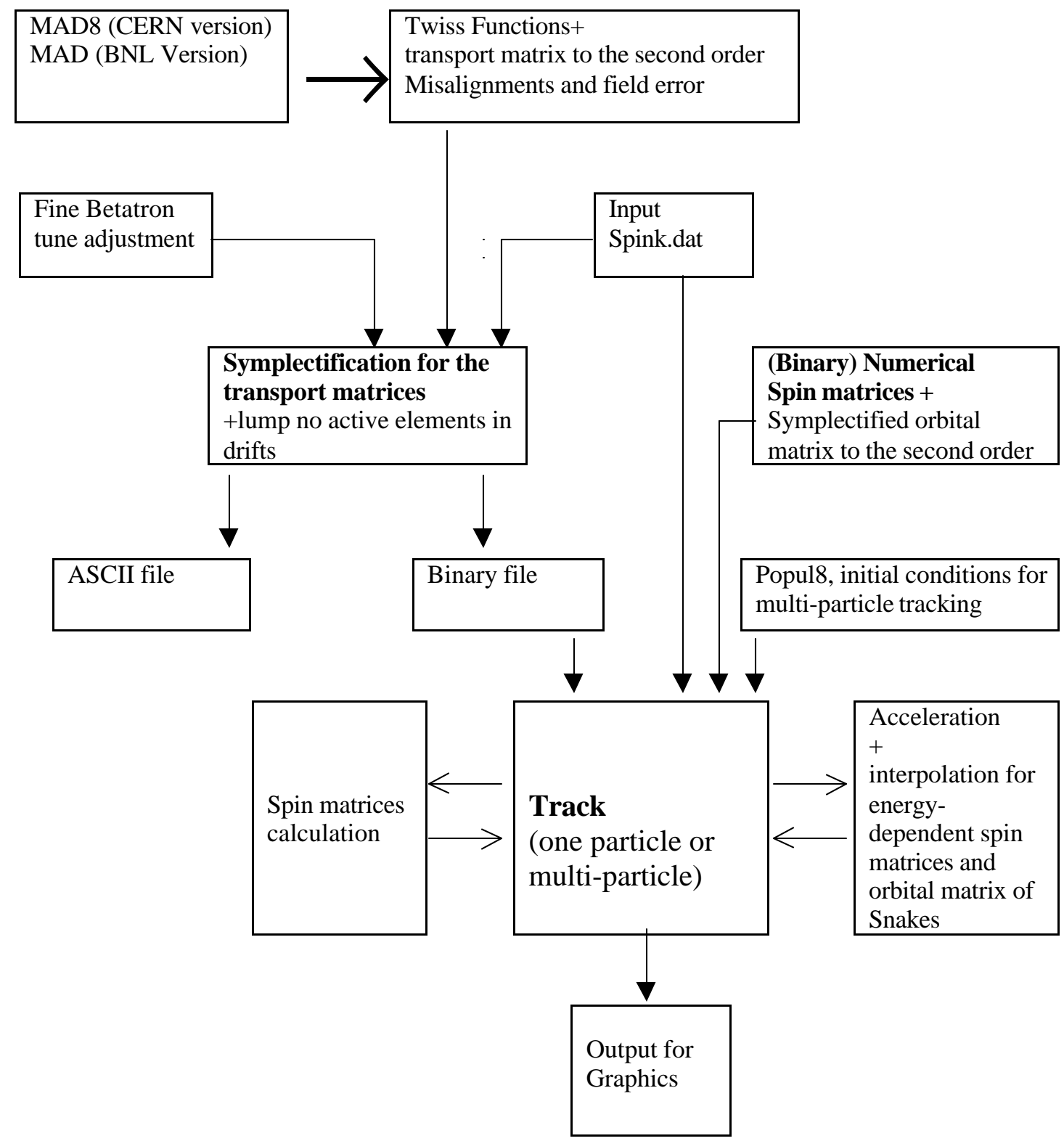

Fig. 1 The logical diagram of Code SPINK 


\subsection{Orbit map for the acceleration of a RF cavity}

Assume the cavity is in a straight section, and is a kick of some sorts, the map for the cavity is

$$
E_{f}=E+F\left(T+T_{o}\right)
$$

where $T=-c \Delta t$ is a phase, $T_{o}$ is the phase of the synchronous particle, $\mathrm{F}()$ is any function. The designed energy changes too

$$
E_{o_{-} f}=E_{o}+F\left(T_{o}\right)
$$

Expression (2.2.1) can be re-arranged as follows:

$$
E_{f}-E_{o}-F\left(T_{o}\right)=\left[E-E_{o}+F\left(T+T_{o}\right)-F\left(T_{o}\right)\right]
$$

Scaled the right side by $p_{s}$, and the left side by $p_{s_{-} f}$, we can get

$$
p_{t_{-} f}=\left(p_{t}+f\left(T+T_{o}\right)-f\left(T_{o}\right)\right) \cdot \frac{p_{s}}{p_{s_{-} f}}
$$

where

$$
f\left(T+T_{o}\right)=\frac{F\left(T+T_{o}\right)}{p_{s} c}
$$

On the other hand, the momenta $p_{x}$ and $p_{y}$ were changed before and after the cavity since the momenta $P_{x}$ and $P_{y}$ must also be re-scaled. We get

$$
\begin{aligned}
& p_{x_{-} f}=p_{x} \cdot\left(\frac{p_{s}}{p_{s_{-} f}}\right) \\
& p_{y_{-} f}=p_{y} \cdot\left(\frac{p_{s}}{p_{s_{-} f}}\right)
\end{aligned}
$$

Suppose the voltage of the thin RF-cavity is

$$
V=\hat{V} \sin \left(\varphi_{s}-\frac{\omega t}{c}\right)=\hat{V} \sin \phi, \quad \phi=\varphi_{s}-\frac{\omega t}{c}
$$


Then the function $f(T)$ in expression (2.2.5) will be

$$
f=\frac{F\left(T+T_{o}\right)}{p_{s} c}=\frac{q V}{p_{s} c}=\frac{q \hat{V}}{p_{s} c} \cdot \sin \phi
$$

Write expression (2.2.4) in a matrix form for the longitudinal motion, we have

$$
\left[\begin{array}{c}
T_{f} \\
p_{t_{-} f}
\end{array}\right]=\left[\frac{f\left(T+T_{o}\right)-f(T)}{T} \cdot \frac{p_{t}}{p_{t_{-} f}} \frac{p_{t}}{p_{t_{-} f}}\right]\left[\begin{array}{c}
T_{o} \\
p_{t_{-} o}
\end{array}\right]
$$

and

$$
\frac{f\left(T+T_{o}\right)-f\left(T_{o}\right)}{T}=\frac{\partial f}{\partial T}=\frac{q \hat{V}}{p_{s} c} \cdot\left(-\frac{\omega}{c}\right) \cos \phi
$$

Finally, we get the 6-dimensional orbital map for the acceleration of a RF-cavity as follows

$$
M=\left[\begin{array}{cccccc}
1 & 0 & & & & \\
0 & \text { factor } & & & & \\
& & 1 & 0 & & \\
& & 0 & \text { factor } & & 0 \\
& & & & 1 & 0 . \text { factor factor }
\end{array}\right]
$$

where $\Omega=\frac{q \hat{V}}{p_{s} c} \cdot\left(\frac{\omega}{c}\right) \cos \phi$, and

$$
\text { factor }=\frac{p_{s}}{p_{s_{-} f}}=\frac{1}{1+d p_{m} / p_{s}}=\frac{1}{1+d(\beta \gamma) /(\beta \gamma)} \approx 1-\frac{d(\beta \gamma)}{\beta \gamma}
$$

$d p_{m}$ is the momentum gain per turn.

Matrix in Eq. (2.2.12) shows obviously that it is not symplectic, let's decompose it into two as follows: 


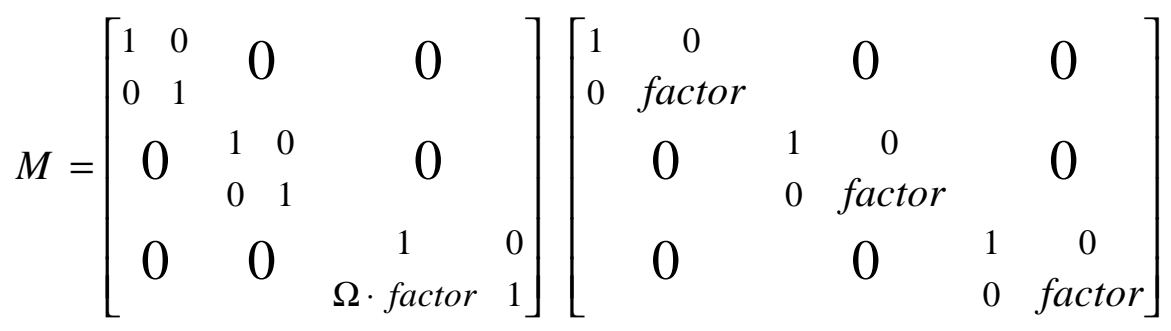

$$
\begin{aligned}
& =M_{\text {symp. }} \circ M_{\text {factor }}
\end{aligned}
$$

Then we can see that $M_{\text {symp. }}$ is a symplectic matrix, and $M_{\text {factor }}$ is somewhat a scale matrix (damping) before and after acceleration. if $d(\beta \gamma) / \beta \gamma$ is small enough, i.e., the acceleration rate is small enough, the tracking can be done up to the top energy without losing the invariants. In RHIC, $d p_{m}$ is about $3 \times 10^{-5}(\mathrm{GeV} / \mathrm{c})$, then $d(\beta \gamma) / \beta \gamma$ is about $10^{-7} \sim 10^{-8}$.

\subsection{The conventions for magnetic field in SPINK}

SPINK uses the same conventions for magnetic field as MAD. The following Taylor expansion for the field on the mid-plane $(y=0)$ was used:

$$
B_{y}(x, 0)=\sum_{n=0}^{\infty} \frac{B_{n} x^{n}}{n !}
$$

The field coefficients have the following meaning:

$B_{o}$, dipole field, with a positive value in the positive $y$ direction; a positive field bends a positively charged particle to the right.

$B_{1}$, quadrupole coefficient $B_{1}=\partial B_{y} / \partial x$; a positive value corresponds to horizontal focussing of a positively charged particle.

$B_{2}$, sextupole coefficient $B_{2}=\partial^{2} B_{y} / \partial x^{2}$.

$B_{3}$, octupole coefficient $B_{3}=\partial^{3} B_{y} / \partial x^{3}$.

The field components can be computed from the longitudinal component of the vector potential to order 4 as: 


$$
\begin{array}{rlr}
B_{x}(x, y)= & +B_{1}\left(y+\frac{h^{2}}{6} y^{3}+\cdots\right) & \\
=B_{2}\left(x y-\frac{h^{3}}{6} y^{3}+\cdots\right) & +B_{3}\left(\frac{1}{6}\left(3 x^{2} y-y^{3}\right)+\cdots\right)+\cdots \\
B_{y}(x, y)=+B_{o} & +B_{1}\left(x-\frac{h}{2} y^{2}+\frac{h^{2}}{2} x y^{2}+\cdots\right) \\
= & B_{2}\left(\frac{1}{2}\left(x^{2}-y^{2}\right)-\frac{h}{2} x y^{2}+\cdots\right)+B_{3}\left(\frac{1}{6}\left(x^{3}-3 x y^{2}\right)+\cdots\right)+\cdots
\end{array}
$$

where $h$ is the curvature of the reference orbit, $h=1 / \rho$

Introducing the magnetic rigidity $B \rho$, the multipole coefficients are computed as

$$
K_{n}=e B_{n} / p_{o}=B_{n} / B \rho
$$

Since the strength of spin resonances depends on the distance of a particle from the equilibrium orbit, the magnetic field strength calculated from the position for the pure dipole, quadrupole, sextupole and multipole were expressed as follows:

Dipole,

$$
\begin{aligned}
& B_{x}=0 . \\
& B_{y}=B_{o}
\end{aligned}
$$

Quadrupole,

$$
\begin{aligned}
& B_{x}=K_{1}(B \rho) y \\
& B_{y}=K_{1}(B \rho) x
\end{aligned}
$$

Sextupole,

$$
\begin{aligned}
& B_{x}=K_{2}(B \rho) x y \\
& B_{y}=K_{2}(B \rho) \cdot \frac{1}{2}\left(x^{2}-y^{2}\right)
\end{aligned}
$$

Multipole,

$$
\begin{aligned}
& B_{x}=K_{1}(B \rho) y+K_{2}(B \rho) x y \\
& B_{y}=K_{1}(B \rho) x+K_{2}(B \rho) \cdot\left(\frac{1}{2}\left(x^{2}-y^{2}\right)\right.
\end{aligned}
$$




\section{Insertion Module for Field-map Generated Matrices of Real Siberian Snakes into RHIC Lattice}

There are two types of Siberian Snakes in each ring of RHIC. Both are supposed to rotate the spin with $180^{\circ}$, but the spin axis is $45^{\circ}$ for Type-A and $-45^{\circ}$ for Type- $\mathrm{B}$, respectively with respect to longitudinal direction $s$ on the $x$-s plane. Two types of real Snakes were introduced into RHIC lattice as follows:

$$
M=\left[D_{L / 2}\right]\left[M_{\text {effect }}\right]\left[D_{L / 2}\right]
$$

where $M$ represents both orbital map $M_{T}$ or spin matrix $M_{S} . M_{T}$ is 6-D symplectified orbital maps of the snakes, $L$ is the length of the Snake, $D_{L / 2}$ is the drift matrix of the half length of the Snake. $M_{\text {effect }}$ is the thin map of the orbit motion and spin precession.

The deviation of the transverse coordinates $\left(x, p_{x}, y, p_{y}\right)$ of the central ray from $(0 ., 0 ., 0$. , 0 .) at the exit of the Snake exists, especially in the low energy region till $100 \mathrm{GeV}$, although the deviations are quite small. They need to be corrected in MAD, together with the lattice misalignments and field errors, by the procedure Micado. The spin tracking for the time being is done by assuming that the deviation has been corrected, and the closed orbit will not be affected by the insertion of the maps of the Snakes.

Since both orbital maps and spin matrices of the Snakes are energy dependent, the interpolation of them at certain energy through the acceleration of polarized proton is needed. The following introduces the interpolation methods to keep the interpolated orbital map symplectic and the interpolated spin matrix unitary, respectively.

\subsection{The interpolation of orbital map up to the second order}

We obtained the symplectic orbital maps of the Snake by extracting the potential candidates : $f$ : for the Poisson bracket operator from a single vector field representation $\vec{F}$ as follows [6]:

$$
\left(M_{k}\right)_{\text {symp. }}=\exp (\vec{F} \cdot \nabla) I d=\exp (: f:) I d
$$

where $\nabla$ is vector field operator, $I d$ is identity map. 
Therefore, we actually stored the Poisson bracket operator :f: at every $0.5 \mathrm{GeV}$ from $25 \mathrm{GeV}$ to $250 \mathrm{GeV}$ in a file, and then, we do the interpolation on :f: at a certain energy $E$ if needed. In this way, the interpolated map can be obtained by expression (3.1.1), and its symplecticity can be guaranteed.

\subsection{The interpolation of spin matrices}

The orthogonal $3 \times 3$ spin matrix consists of 9 elements. The spin matrix at the energy $E$ between $E_{\mathrm{i}}$ and $E_{\mathrm{i}+1}(i=1,2, \ldots, n)$ could be interpolated simply by interpolating each of the element of the spin matrices at $E_{\mathrm{i}}$ and $E_{\mathrm{i}+1}\left(E_{\mathrm{i}+1}-E_{\mathrm{i}}=\Delta E\right.$, a constant energy $)$. However, it can be shown that the unitarity of the interpolated spin matrices obtained in this way can not be guaranteed. It results in spin polarization totally lost after 10,000 turns of spin tracking.

Note that the rotation of the spin is a subgroup of the space rotation $\mathrm{SO}(3)$. The spin matrix can be described completely using a smaller number of parameters, the three components of vector $\vec{v}=\left(v_{1}, v_{2}, v_{3}\right)$ as follows [8]:

$$
M_{s}=\left[\begin{array}{lll}
s_{11} & s_{12} & s_{13} \\
s_{21} & s_{22} & s_{23} \\
s_{31} & s_{32} & s_{33}
\end{array}\right]=\exp (-\theta \vec{\omega} \cdot \vec{J})=\exp (\vec{v} \cdot \vec{J})
$$

where $J_{i}$ are $3 \times 3$ matrices as follows:

$$
J_{1}=\left[\begin{array}{ccc}
0 & 0 & 0 \\
0 & 0 & 1 \\
0 & -1 & 0
\end{array}\right], J_{2}=\left[\begin{array}{ccc}
0 & 0 & -1 \\
0 & 0 & 0 \\
1 & 0 & 0
\end{array}\right], J_{2}=\left[\begin{array}{ccc}
-1 & 0 & 0 \\
0 & 0 & 0 \\
0 & 0 & 1
\end{array}\right]
$$

The interpolation for the spin matrix on vector $\vec{v}$ can be done as follows: First, by calculating the eigenvalues and eigenvectors of the matrix of $M_{s}\left(E_{i}\right)$, we obtain the rotation angle $\theta$ and nomalized eigaivector $\vec{\omega}$. Therefore, $\vec{v}=\theta \vec{\omega}$. We write and store the three components of the vector

$$
\vec{v}\left(E_{i}\right)=\left[v_{1}\left(E_{i}\right), v_{2}\left(E_{i}\right), v_{3}\left(E_{i}\right)\right], \quad i=1,2, \ldots . n
$$


at energy $E_{1}, E_{2}, \ldots, E_{n}$ from the injection energy of $25 \mathrm{GeV}$ to the top energy of $250 \mathrm{GeV}$ with uniform step $\Delta E$. Then, we interpolate the three elements of the vector $\vec{v}$ at the energy $E$, which is between $E_{\mathrm{i}}$ and $E_{\mathrm{i}+1}$. Finally, the spin matrix can be recalculated by Eq. (3.2.1) and its unitarity is thus guaranteed.

\section{Symplectification of the orbit maps provided by MAD}

\subsection{Symplectic Condition Checking}

To evaluate the spin precession of polarized proton in RHIC, orbital tracking will be done with the orbital maps of all the elements in RHIC lattice, except Siberian Snakes, output directly from MAD. Therefore, the symplecticity has to be checked for these maps before they are used in long-term tracking.

The maximum symplecticity violation of $9.57 \times 10^{-8}$ is found for the map of a quadrupole, Q106, in RHIC lattice. The One Turn Map (OTM) can be obtained by concatenating every matrix, except Siberian snakes in the ring as follows:

$$
O T M=\left[\begin{array}{cccccc}
\underline{3.49 D-01} & \underline{9.29 D+00} & 0.00 D+00 & 0.00 D+00 & 0.00 D+00 & \underline{-4.14 D-02} \\
\underline{-9.24 D-02} & \underline{3.56 D-01} & 0.00 D+00 & 0.00 D+00 & 0.00 D+00 & \underline{2.89 D-03} \\
0.00 D+00 & 0.00 D+00 & \underline{4.36 D-01} & \underline{9.04 D+00} & 0.00 D+00 & 0.00 D+00 \\
0.00 D+00 & 0.00 D+00 & \underline{-8.91 D-02} & \underline{4.45 D-01} & 0.00 D+00 & 0.00 D+00 \\
\underline{2.89 D-03} & \underline{-4.16 D-02} & 0.00 D+00 & 0.00 D+00 & \underline{1.00 D+00} & \underline{-7.36 D+00} \\
0.00 D+00 & 0.00 D+00 & 0.00 D+00 & 0.00 D+00 & 0.00 D+00 & \underline{1.00 D+00}
\end{array}\right]
$$

The result of the symplecticity condition checking for OTM is given as follows.

$$
O T M=\left[\begin{array}{cccccc}
\underline{3.49 D-01} & \underline{9.29 D+00} & 0.00 D+00 & 0.00 D+00 & 0.00 D+00 & \underline{-4.14 D-02} \\
\underline{-9.24 D-02} & \underline{3.56 D-01} & 0.00 D+00 & 0.00 D+00 & 0.00 D+00 & \underline{2.89 D-03} \\
0.00 D+00 & 0.00 D+00 & \underline{4.36 D-01} & \underline{9.04 D+00} & 0.00 D+00 & 0.00 D+00 \\
0.00 D+00 & 0.00 D+00 & \underline{-8.91 D-02} & \underline{4.45 D-01} & 0.00 D+00 & 0.00 D+00 \\
\underline{2.89 D-03} & \underline{-4.16 D-02} & 0.00 D+00 & 0.00 D+00 & \underline{1.00 D+00} & \underline{-7.36 D+00} \\
0.00 D+00 & 0.00 D+00 & 0.00 D+00 & 0.00 D+00 & 0.00 D+00 & \underline{1.00 D+00}
\end{array}\right]
$$

The result of the symplecticity condition checking for OTM is given as follows. 
$(\mathrm{OTM})^{\mathrm{T}} \cdot \mathrm{J} \cdot(\mathrm{OTM})-\mathrm{J}=$

$$
\left[\begin{array}{cccccc}
0.00 D+00 & \underline{-5.60 D-06} & 0.00 D+00 & 0.00 D+00 & 0.00 D+00 & \underline{3.14 D-08} \\
\underline{5.60 D-06} & 0.00 D+00 & 0.00 D+00 & 0.00 D+00 & 0.00 D+00 & \underline{-4.64 D-08} \\
0.00 D+00 & 0.00 D+00 & 0.00 D+00 & \underline{-4.46 D-09} & 0.00 D+00 & 0.00 D+00 \\
0.00 D+00 & 0.00 D+00 & \underline{4.46 D-09} & 0.00 D+00 & 0.00 D+00 & 0.00 D+00 \\
0.00 D+00 & 0.00 D+00 & 0.00 D+00 & 0.00 D+00 & 0.00 D+00 & 0.00 D+00 \\
\underline{3.14 D-08} & \underline{4.64 D-08} & 0.00 D+00 & 0.00 D+00 & 0.00 D+00 & 0.00 D+00
\end{array}\right]
$$

Therefore the symplecticity violation in $\left(x, p_{x}\right)$ phase space is $5.6 \times 10^{-6}$, in $\left(y, p_{y}\right)$ phase space is $4.46 \times 10^{-9}$. Obviously, it is not accurate enough for the tracking over $10^{7}$ turns.

\subsection{Exponential and Cayley Symplectifications}

We know that a matrix $\mathrm{M}$ can be written in the exponential form [9]

$$
M=\exp (B)
$$

where $B$ is a real matrix. Then we may write the relations

$$
\begin{aligned}
M & =\exp (B)=[\exp (B / 2) / \exp (-B / 2)] \\
& =[\cosh (B / 2)+\sinh (B / 2)] /[\cosh (B / 2)-\sinh (B / 2)] \\
& =[I+\tanh (B / 2)] /[I-\tanh (B / 2)] .
\end{aligned}
$$

Define a matrix $T$ by the equation

$$
T=\tanh (B / 2)
$$

With the aid of $T, M$ as given by (4.2.2) has the Cayley representation

$$
M=(I+T)(I-T)^{-1}=(I-T)^{-1}(I+T),
$$

Relation (3.2.2) can be solved for $T$ to give the result

$$
T=(M+I)(M-I)^{-1}=(M-I)(M+I)^{-1},
$$

Define the matrix $V$ by the equation 


$$
V=J^{-1} T
$$

$J$ is the matrix described in Eq. (1.4.3). We know that $V$ will be symmetric if $M$ is symplectic, and vice versa. Consequently, $V$ will be nearly symmetric if $M$ is nearly symplectic. Let us define a symmetric matrix $W$ by taking the symmetric part of $V$,

$$
W=\left(V+V^{T}\right) / 2
$$

then we may define a symplectic matrix $R$ by writing

$$
R=(I+J W)(I-J W)^{-1}=(I-J W)^{-1}(I+J W),
$$

and $R$ will be a symplectification of $M$ that we call the Cayley symplectification.

The first order matrices of the elements in RHIC lattice are symplectified by the method described above, and symplecticity of the matrices will be up to machine (computer) precision.

\section{Validation of the Theory}

Orbital transfer maps are made symplectic before being used to do the tracking. Then, the following quantities, in particular the beam oscillation normalized amplitudes will remain constant during acceleration, if the orbital motions are linear and without coupling.

$$
\begin{aligned}
& x_{\text {amp }}=\sqrt{\beta_{T_{-} x} \varepsilon_{N_{-} x}}=\sqrt{\left(x^{2}+p_{x n}^{2}\right) \cdot(\beta \gamma)} \\
& y_{\text {amp }}=\sqrt{\beta_{T_{-} y} \varepsilon_{N_{-} y}}=\sqrt{\left(y^{2}+p_{y n}^{2}\right) \cdot(\beta \gamma)}
\end{aligned}
$$

where

$$
\begin{aligned}
& p_{x n}=x^{\prime} \beta_{T_{-} x}+x \boldsymbol{\alpha}_{T_{-} x} \\
& p_{y n}=y^{\prime} \beta_{T_{-} y}+y \boldsymbol{\alpha}_{T_{-} y}
\end{aligned}
$$

and $\boldsymbol{\beta}_{T_{-} x}, \boldsymbol{\alpha}_{T_{-} x}, \boldsymbol{\beta}_{T_{-} y}, \boldsymbol{\alpha}_{T_{-} y}$ are Twiss functions of the lattice, $\boldsymbol{\varepsilon}_{N_{-} x}, \boldsymbol{\varepsilon}_{N_{-} y}$ are normalized emittances in $x, y$ direction. We took $x_{a m p}, y_{\text {amp }}$ to validate the invariants during the acceleration. 
Actually, the Siberian Snakes introduce transverse coupling into the orbital motion. However, there still exist three invariants in $x, y$ and $s$ phase space since all the orbital maps are symplectic. We make the assumption that all eigenvalues $\lambda_{\mathrm{k}}$ of a real symplectic matrix $M$ are distinct. This implies that the eigenvectors $v_{k}$ are all linearly independent and form a basis in $2 N$ - dimensional space. Since the transfer matrix is real, the eigenvalues and eigenvectors form complex conjugate pairs. Decomposing the eigenvalues $\lambda_{k}$ and eigenvectors $v_{k}$ into their real and imaginary parts[10]:

$$
\begin{gathered}
v_{k}=a_{k} \pm i b_{k} \quad \lambda_{k}=\cos \mu_{k} \pm i \sin \mu_{k} \\
(k=1, \quad \cdots \quad N)
\end{gathered}
$$

For stable motion, the tune $\mu_{k}$ are real. The three tunes $\mu_{k}(k=1,2,3)$ by each One Turn Map will be calculated to see the effects of the real Siberian Snakes, inserted in the form of numerical matrix, on the betatron oscillation.

\section{Spin Tracking with "Real” Siberian Snakes in RHIC}

In this section, if a single particle is tracked, it is extracted from the contour of phase space with the normalized r.m.s emittances of $5 \pi \mathrm{mm} \cdot \mathrm{mrad}, 10 \pi \mathrm{mm} \cdot \mathrm{mrad}, 15 \pi \mathrm{mm} \cdot \mathrm{mrad}$ and $20 \pi \mathrm{mm} \cdot \mathrm{mrad}$, respectively. In a multi-particle simulation, particles are extracted from the inside of the phase space volume randomly with a Gaussian distribution. Each particle is tracked with acceleration in RHIC from the injection energy of $25 \mathrm{GeV}$ to the top energy of $250 \mathrm{GeV}$. The momentum gain per turn is $3 \times 10^{-5}(\mathrm{GeV} / \mathrm{c})$. This value was obtained by imitating the actual operation mode in RHIC as follows: There are 5 RF cavities in each RHIC ring, while only two of them will be used for the acceleration of polarized proton with the peak voltage $\sim 300 \mathrm{KV}$ in 80 second. Totally about $7.5 \times 10^{6}$ turns has to be tracked. In this section, the first order of the orbital map was used after proper symplectification.

\subsection{Spin tracking with Perfect Siberian Snakes in RHIC with and without symplectification of the orbital maps}


The orbital map of perfect Siberian Snakes was set to be identity, and the spin rotation matrices for Snake Type-A and Snake Type-B are given as follows [11],

- Type-A: $\theta=\pi / 4, \phi=0, \psi=\pi$, i.e., spin axis is in the $\mathrm{x}$-s plane, beam direction is in s- direction, then

$$
M_{s}=\left[\begin{array}{ccc}
0 & 0 & 1 \\
0 & -1 & 0 \\
1 & 0 & 0
\end{array}\right]
$$

- Type-B: $\theta=-\pi / 4, \phi=0, \psi=\pi$, i.e., the spin axis is in the $\mathrm{x}$-s plane, the beam direction is in s-direction, then

$$
M_{s}=\left[\begin{array}{ccc}
0 & 0 & -1 \\
0 & -1 & 0 \\
-1 & 0 & 0
\end{array}\right]
$$

Fig. $2 \mathrm{a}$ and Fig. $2 \mathrm{~b}$ give the spin tracking results of polarized protons without doing symplectification for the orbital maps of the elements in RHIC lattice. The particle in Fig. $2 a$ was extracted from the contour of $5 \pi \mathrm{mm}$.mrad, and the particle in Fig. $2 b$ from the contour of $20 \pi \mathrm{mm}$.mrad. The normalized amplitudes are recorded since we suppose perfect Siberian Snakes do not introduce the transverse coupling. Both figures show that the normalized amplitude in $x$ phase space decreases with the acceleration. It means that the normalized emittance in $x$ was shrunk artificially. This is identical to the result of symplecticity checking, since the symplecticity violation of the one turn map is $5.6 \times 10^{-6}$ in $x$-direction, but $4.46 \times 10^{-9}$ in $y$-direction. Therefore, the spin depolarization results in both cases may be wrong.

Fig. $3 \mathrm{a}$ and Fig. $3 \mathrm{~b}$ give the spin tracking results with the symplectified orbital maps for the particle from a contour of $5 \pi \mathrm{mm} . \mathrm{mrad}$ and $20 \pi \mathrm{mm}$.mrad. The figures show that the normalized amplitudes are kept constant through the whole acceleration range. We can see that the spin depolarization results are different from those without doing symplectification. Fig. 4 gives the spin tracking results of $5 \pi \mathrm{mm} . \mathrm{mrad}, 10 \pi \mathrm{mm} . \mathrm{mrad}$, $15 \pi \mathrm{mm}$.mrad and $20 \pi \mathrm{mm}$.mrad. We know that the intrinsic resonances are enhanced due 
to the $P$ superperiods at $K=m P \pm v_{\mathrm{y}}$ and the $M$ FODO cells at $K=m P M \pm v_{\mathrm{B}}$, where $v_{\mathrm{B}}$ is the total accumulated phase advance in dipole cells. But the enhancement due to $M$ is much more important because normally $M>>P$ in high energy accelerators. Since $m P M \pm v_{\mathrm{B}}$ may not necessarily coincide with the resonance condition of $m P \pm v_{\mathrm{y}}$, important resonances occur at those $K=n P \pm v_{\mathrm{y}}$ such that they are closest to $m P M \pm v_{\mathrm{B}}$. Furthermore, dominant resonances are located at $m=$ odd integers, since where spin kicks due to focusing and defocusing quadrupoles add up coherently. We found in Fig. 4 that three strong depolarization resonances happened at $(G \gamma)_{1}=3 \times 81-\left(v_{y}-12\right)=225.82,(G \gamma)_{2}=$ $3 \times 81+\left(v_{y}-12\right)=260.18$, and $(G \gamma)_{4}=5 \times 81+\left(v_{y}-12\right)=422.18$, where 81 is the product of superperiodicity $P=3$ and the "effective" FODO cells per superperoid $M=27$, which includes dispersion suppressors in RHIC. $2 \pi v_{\mathrm{B}}=2 \pi\left(v_{\mathrm{y}}-12\right)$ is the accumulated phase advance of all FODO cells. Another strong depolarizing resonance has found at the location of $(G \gamma)_{3}=5 \times 81-\left(v_{y}-6\right)=381.82$.

\subsection{Spin tracking with "real" Siberian Snakes in RHIC in the presence of intrinsic and imperfection resonances}

\subsubsection{In the presence of intrinsic resonances}

The spin tracking of polarized proton was carried out with "real" Siberian Snakes using symplectifed numerical orbit maps and spin matrices for the two types of Snakes in RHIC lattice. Fig. 5 gives the single particle tracking results in a RHIC machine with no misalignment and no field error for the particle extracted from the contour of $5 \pi \mathrm{mm} . \mathrm{mrad}, 10 \pi \mathrm{mm} . \mathrm{mrad}, 15 \pi \mathrm{mm} . \mathrm{mrad}$ and $20 \pi \mathrm{mm} . \mathrm{mrad}$, respectively. Then, the depolarization resonances shown in these figures are only intrinsic resonances.

Comparing with results in Fig. 4, we find that "real" snakes can maintain the polarization of the proton better than the perfect snakes in RHIC. In the case of perfect snakes in RHIC, the depolarization can not be recovered completely after $(G \gamma)_{3}=5 \times 81-\left(v_{\mathrm{y}}-\right.$ $6)=381.82$ for the particle from $10 \pi \mathrm{mm}$.mrad and the final polarization is kept to $88 \%$. On the other hand, the polarization can be kept to $95 \%$ at the top energy $(\mathrm{G} \gamma=477)$ in the case of "real" snakes. 
We recorded the betatron amplitudes in $x$ and $y$ directions at $6-\mathrm{o}^{\text {'clock (the starting }}$ point of the RHIC ring, $\left.\beta^{*} \mathrm{~T}_{-} \mathrm{x}=9.956 \mathrm{~m}, \beta^{*} \mathrm{~T}_{-\mathrm{y}}=10.044 \mathrm{~m}\right)$ in the ring for perfect snake and "real" snake cases, shown in Fig. 6a and Fig. 6b, respectively. The particle is extracted from the emittance of $\varepsilon_{\mathrm{x}}=\varepsilon_{\mathrm{y}}=5 \pi \mathrm{mm}$.mrad. The $x$ and $y$ amplitudes decrease with the acceleration due to the adiabatic damping effect. In Fig. $6 b$ the amplitude in $y$ direction is getting smaller, and $x$ direction is getting larger comparing to the betatron oscillations in Fig. 6a where amplitudes in $x$ and $y$ directions are the same values throughout the acceleration. We also found that the amplitudes oscillated strongly at low energy region $(G \gamma 150)$ for the "real" snake case. It is because the "real" Siberian Snakes in the ring introduce the transverse coupling effects in betatron motions.

These coupling effects may result in the change of actual tune $Q_{\mathrm{x}}$ and $Q_{\mathrm{y}}$ change. We calculated these tunes from the eigenvalues of the One Turn Map (OTM), which was concatenated by the matrix of each element in the lattice, including two Siberian Snakes' maps at a certain energy. Fig. 7 gives the betatron tunes in $x$ and $y$ direction varied with the acceleration due to the insertion of the Snakes. We can see that they decrease with the energy of the polarized proton. The maximum tune shifts from the designed working points are $\Delta Q_{\mathrm{x}}=0.01919, \Delta Q_{\mathrm{y}}=0.0306$ at injection energy $25 \mathrm{GeV}$. Fig. 8 is the tune diagram showing the selected working point in RHIC at $Q_{\mathrm{x}}=28.19$ and $Q_{\mathrm{y}}=29.18$, and the neighboring sum resonances $n Q_{\mathrm{x}}+m Q_{\mathrm{y}}=p$, where $n, m$ and $p$ are positive integers and $n+m$ denotes the order of the resonances, as well as difference resonance $Q_{\mathrm{x}}-Q_{\mathrm{y}}=-1$. The usable tune range in RHIC is 0.034 , as the nominal tune is located between the $5^{\text {th }}$ order resonance at 28.20 and the $6^{\text {th }}$ order resonance at 28.166. It also indicates that the working point with "real" Siberian Snakes moves to the selected working point of RHIC. We can see that it crosses $5^{\text {th }}$ and $10^{\text {th }}$ order sum resonance lines when the energies are less than $37.5 \mathrm{GeV}(G \gamma=71.67)$, causing transverse coupling, which may result in a large amplitude of betatron oscillation in both $x$ and $y$ directions. In the region of energies from $45 \mathrm{GeV}$ to $70 \mathrm{GeV}$ ( $G \gamma$ from 86.01 to 133.79), the working point crosses difference resonance $Q_{\mathrm{x}}-Q_{\mathrm{y}}=-1$. Since difference resonances only cause the phase space topology distorted, the system is still kept stable.

We found that the strong resonance strengths in the low energy region are about 3 factors smaller than those in the high-energy region. Therefore, the spin motion was not so 
seriously affected by the large betatron oscillation amplitude in the low energy region due to the transverse coupling.

\subsubsection{In the presence of both intrinsic and imperfection resonances}

Spin tracking was also done in a RHIC machine with misalignments. The misalignments of each magnet in all the FODO arc cells of the lattice were assigned randomly in MAD by truncated Gaussian distribution functions. To study the effect of lattice misalignments as well as the direct and indirect effect of the closed orbit correctors on spin motion, the simulation was carried out in two groups.

\section{Group 1:}

The standard deviations of the truncated Gaussian distribution functions for the misalignment assignment of main dipoles are $1 \mathrm{mrad}$ only for the rotation angle around the $s$-axis, and for those of the main quadrupoles (QF, QD) and sextupoles are $1 \mathrm{~mm}$ for the positions in $x$-direction and $y$-direction. The r.m.s. value $(\sigma)$ in Gaussian distribution function is set to be $0.1 \mathrm{~mm}, 0.5 \mathrm{~mm}, 1.0 \mathrm{~mm}$ and $2.5 \mathrm{~mm}$, respectively. The closed orbit distortions were all corrected to about $0.180 \mathrm{~mm}$ (r.m.s) using Micado, and the tune $\mathrm{Q}_{\mathrm{x}}$ and $\mathrm{Q}_{\mathrm{y}}$ were kept to be 28.19 and 29.18, respectively by adjusting the quadrupoles' strengths of the arc cells. The single-particle tracking was done for the particle extract from zero emittance $\varepsilon_{\mathrm{x}}=\varepsilon_{\mathrm{y}}=0$. In this case, the imperfection resonances play the main role in spin depolarizing resonances. The tracking results are given in Fig. 9 (a), (b), (c) and (d), respectively. It was shown in these figures that the bigger the misalignments, the stronger the depolarization. It reflects the direct and local effect of the correctors in the lattice on spin motion. If the misalignments are bigger, the magnetic field strengths of the correctors should be stronger to correct the closed orbit to the same value. Therefore, the transverse perturbation radial fields arising from the correctors on spin are getting larger.

\section{Group 2:}

The standard deviations of the truncated Gaussian distribution functions for the misalignment assignment of main dipoles are $1 \mathrm{mrad}$ only for the rotation angle around the $s$-axis, and for those of the main quadrupoles (QF, QD) and sextuples are $1 \mathrm{~mm}$ for the positions in $x$-direction and $y$-direction. The r.m.s. value $(\sigma)$ in Gaussian distribution 
function is set $2.5 \mathrm{~mm}$. By using the procedures of Micado in MAD, the vertical closed orbit distortion was corrected to about $0.189 \mathrm{~mm}$ (r.m.s.) and $0.512 \mathrm{~mm}$ (r.m.s.). The tune $\mathrm{Q}_{\mathrm{x}}$ and $\mathrm{Q}_{\mathrm{y}}$ were kept to be 28.19 and 29.18 , respectively in both cases by adjusting the quadruples' strengths of the arc cell. Since the strength of spin resonances depends on the distance of a particle from the central orbit, the depolarization affected by lattice errors can be simulated in this way. The single particle spin tracking was done for the particle from the emittance of $5 \pi \mathrm{mm} . \mathrm{mrad}, 10 \pi \mathrm{mm} . \mathrm{mrad}, 15 \pi \mathrm{mm} . \mathrm{mrad}$ and $20 \pi \mathrm{mm} . \mathrm{mrad}$, respectively. The results are shown for corrected closed orbit distortion of $0.189 \mathrm{~mm}$ in Fig 4.10 and $0.512 \mathrm{~mm}$ in Fig 4.11, respectively. The depolarization resonances shown in these figures are getting larger in the presence of both intrinsic and imperfection resonances. We know that the tolerable imperfection resonance strength decreases dramatically due to the overlapping effect of two types of resonances[12]. In case of the closed orbit distortion corrected to within $0.189 \mathrm{~mm}$ (r.m.s.), the imperfection resonance strength is less than 0.07 , about 6 times less than the intrinsic resonance strength, the polarization can still be kept to $88 \%$ if the polarized proton is extracted from a contour of $10 \pi \mathrm{mm} \cdot \mathrm{mrad}$. On the other hand, when the corrected closed orbit distortion is $0.5 \mathrm{~mm}$, the polarization can be kept to only $36 \%$ even though the polarized proton is extracted from a contour of $5 \pi \mathrm{mm} \cdot \mathrm{mrad}$, and to $44 \%$ for $10 \pi \mathrm{mm} \cdot \mathrm{mrad}$ case.

\subsection{Multi - particle tracking results}

Multi - particle tracking is performed with 32 particles generated from the inside of the phase space volume randomly with a Gaussian distribution. We observe from single particle tracking results that two strong depolarization resonances happen at $G \gamma=381.82$ and $G \gamma=422.18$. Therefore, the particles are tracked with acceleration in RHIC from $197.92 \mathrm{GeV}(G \gamma=378.18)$ to the top energy $250 \mathrm{GeV}(G \gamma=477.67)$, for 32 particles extracted from the emittance of $10 \pi \mathrm{mm} . \mathrm{mrad}$.

The average polarization was calculated in two ways as follows:

$$
\begin{aligned}
& S_{y_{-} \text {average }}=\frac{1}{N} \sum_{N} S_{y} \\
& S_{y_{-} \text {average }}(\text { r.m.s. })=\frac{1}{N} \sqrt{\sum_{N} S_{y}^{2}}
\end{aligned}
$$


where $N$ is the number of particles, $S_{y}$ is the polarization of each particle during the acceleration. Firstly, we have tracked with perfect Siberian Snakes and with "real" Siberian Snakes in a RHIC machine with no lattice misalignment. Fig. 12a and Fig. 12b indicate the average polarization $\mathrm{S}_{\mathrm{y}_{\_} \text {average }}$ and $\mathrm{S}_{\mathrm{y}_{\_} \text {average }}$ (r.m.s) of 32 particles in two cases respectively. It was found that the strong depolarizing resonances happen around $G \gamma=$ $411-v_{\mathrm{y}}=381.82$ and $451-\mathrm{v}_{\mathrm{y}}=421.82$. The perfect snakes can keep the spin polarization up to $88.86 \%$ for average and $87.67 \%$ for r.m.s. value at the top energy 250 $\mathrm{GeV}$, but "real" Siberian Snakes can keep the polarization up to $96.25 \%$ for average and $94.65 \%$ for r.m.s value. These results are consistent to those of single-particle tracking. We also tracked in a RHIC machine with r.m.s. lattice misalignments of $2.5 \mathrm{~mm}$ and the vertical corrected closed orbit distortion of $0.189 \mathrm{~mm}$. The average polarization $\mathrm{S}_{\mathrm{y}_{\mathrm{a}} \text { average }}$ and $S_{y_{-} \text {average }}$ (r.m.s) are given in Fig. 13. It was found that the spin polarization could be kept up to $63.06 \%$ for average and $73.00 \%$ for r.m.s. value for the real Snakes.

\section{Summary}

The spin tracking with "real" Siberian Snakes in RHIC has been performed in the presence of intrinsic and imperfection resonances. We summarize the results as follows

(1) Single particle tracking shows that "real" Siberian Snakes can keep the polarization of the protons through the acceleration to more than $95 \%$ in a RHIC machine with no misalignment and no field error, if the polarized proton is extracted from a contour of $10 \pi \mathrm{mm} . \mathrm{mrad}$; Since the closed orbit correctors has direct and indirect effect on spin motion in the ring, we found that the bigger the misalignments, the stronger the depolarization resonances; In the case that the misalignment (r.m.s.) is $2.5 \mathrm{~mm}$, "real" Siberian Snakes can keep the polarization of the proton to more than $88 \%$ in a machine with corrected vertical closed orbit distortion (r.m.s.) of $0.189 \mathrm{~mm}$, if the polarized proton is extracted from a contour of $10 \pi \mathrm{mm} . \mathrm{mrad}$; But the polarization is only $38 \%$ when the polarized proton is extracted from a contour of $5 \pi \mathrm{mm}$.mrad in the case that corrected closed orbit distortion is $0.512 \mathrm{~mm}$ (r.m.s). 
(2) Multi-particle tracking shows that "real" Siberian Snakes can keep the polarization of the protons to $73.00 \%$ (r.m.s.) through the acceleration in a RHIC machine with r.m.s. misalignment of $2.5 \mathrm{~mm}$ and corrected vertical closed orbit distortion of $0.189 \mathrm{~mm}$ (r.m.s), if the polarized protons are extracted from a contour of $10 \pi \mathrm{mm} . \mathrm{mrad}$.

(3) The symplectic problem on the orbital motion is one of the key issues for spin tracking. We have solved this problem for the "real" Siberian Snakes to predict the "actual” operation conditions in RHIC.

(4) Due to the presence of large longitudinal magnetic fields in "real" Snake, the coupling of horizontal and vertical betatron motions are found, and the vertical betatron tune is found to change from 29.22 at the injection energy $25 \mathrm{GeV}$ to 29.18 at the top energy $250 \mathrm{GeV}$. It is found that the operation points cross four $10^{\text {th }}$ order sum resonances lines, four $5^{\text {th }}$ order sum resonance lines and one difference resonance line during the acceleration. At these resonance crossings, the betatron amplitudes rapidly oscillate, and the vertical amplitude becomes larger due to the coupling effects. However, these motions in the low energy region do not affect so seriously the spin polarization.

\section{Acknowledgments}

This work was done with the members of spin group of AGS at BNL, supported by RHIC-spin project. I (Meiqin Xiao) would like to thank Dr. Thomas Roser for his invitation and arrangement. The discussion with him made me understood the symplectic problem is not only for the numerical maps of Siberian Snakes themselves, but also for the maps of all the other elements in RHIC lattice. I would like to thank Dr. Alfredo Luccio for providing code SPINK to me and for his kindly cooperation with me. I learned a lot from him on orbit and spin dynamics, as well as computer operation. I appreciated Dr. Nick Tsoupas for his constant help, his detailed experience saved me a lot of time. I also want to thank Dr. W. Mackay, Dr. A Lehrach, Dr. H. Huang and Dr. M. Bai for their useful discussion. Dr. S. Tepikian, Dr. W. Fischer and the people in their group helped me to settle down at BNL. Mr. K. Brown helped me to solve computer problem. The secretary Ms. M. Campbell helped me constantly during my staying at BNL. 


\section{Reference}

[1] A.luccio, Numerical Spin Tracking in a Synchrotron. Computer Code SPINK. Examples(RHIC). Brookhaven National Laboratory, AGS/RHIC/SN No. 011.

[2] H. Wu, et al., The Spin Tracking Study in RHIC for Polarized Proton. Brookhaven National Laboratory, AGS/RHIC/SN No. 026.

[3] J. P. Blewett and R. Chasman, J. Ap. Physics., 48(1977)2692.

[4] A. U. Luccio, Polarized Protons Tracking in the AGS and RHIC. Submitted to Polarized Protons at High Energies-- Accelerator Challenges and Physics Opportunities Workshop.BNL,1999.

[5] M.Xiao and T. Katayama, The Simulation of Siberian Dnakes based on Calculated Three Dimensional Magnetic Field. BNL Spin Note AGS/RHIC/SN No.70, December 3,1997;

[6] M.Xiao, et al, DA(Differential Algebraic) Method and Symplectification for Field map Generated Matrices of Siberian Snakes, BNL Spin Note AGS/RHIC/SN No.78, September 10,1998; Proceedings of the 1999 Particle Accelerator Conference, New York City, March 29th - April 2nd, 1999.

[7] F.Christoph Iselin, The MAD Program Physical Methods Manual. Geneva, Switzerland. January 10, 1994.

[8] E. Corinaldes, Classical Mechanics for Physics Graduate Students. Chapter 10, Relativistic Dynamics, $\mathrm{P}_{227 \sim 259}$.

[9] A.J. Dragt, Lectures on Nonlinear Dynamics and Lie Methods with Applications to Accelerator Physics. Chapter 4, $\mathrm{P}_{206}$. Center for theoretical Physics, Department of physics, university of Maryland. 1998.

[10] L. C. Teng, Concerning n-Dimensional Coupled Motion. FN 229, FNAL,1971.

[11] T. Katayama, Idealistic Spin Transfer Matrix, Personal report, 1997.

[12] S.Y. Lee, Prospects for Polarization at RHIC and SSC, Polarized Collider Workshop, AIP Conference Proceeding Vol. 223, $\mathrm{P}_{30}, 1990$. 


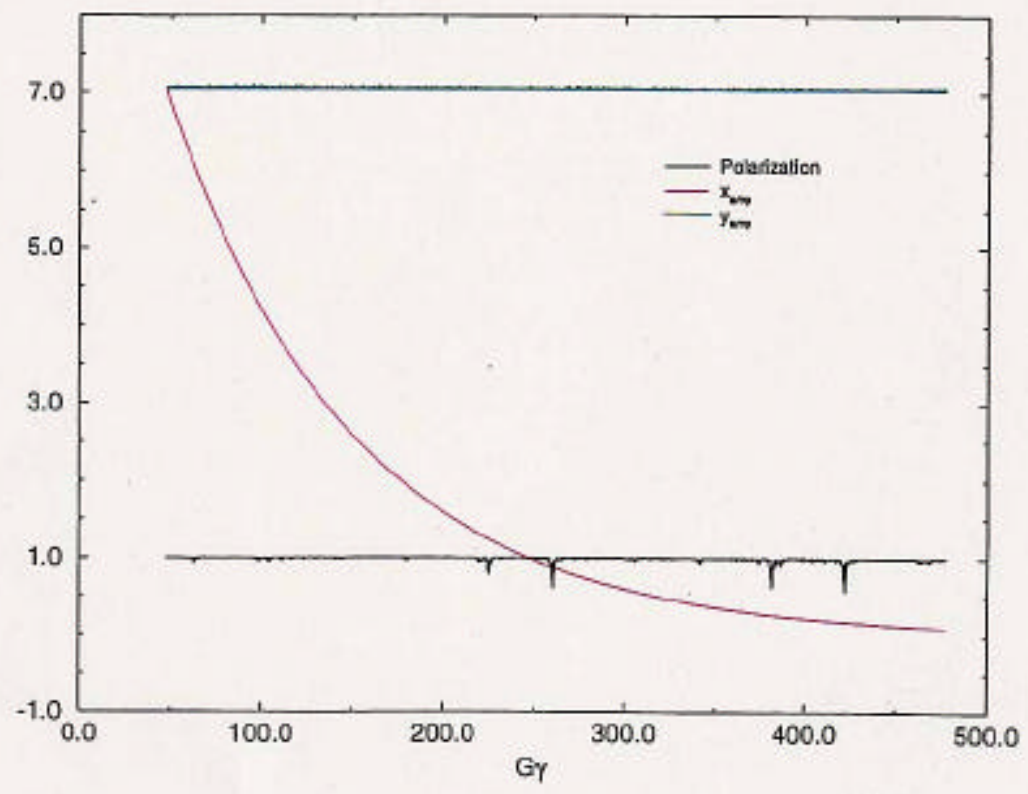

a. $\varepsilon_{\mathrm{x}}=\varepsilon_{\mathrm{y}}=5 \pi \mathrm{mm} \cdot \mathrm{mrad}$

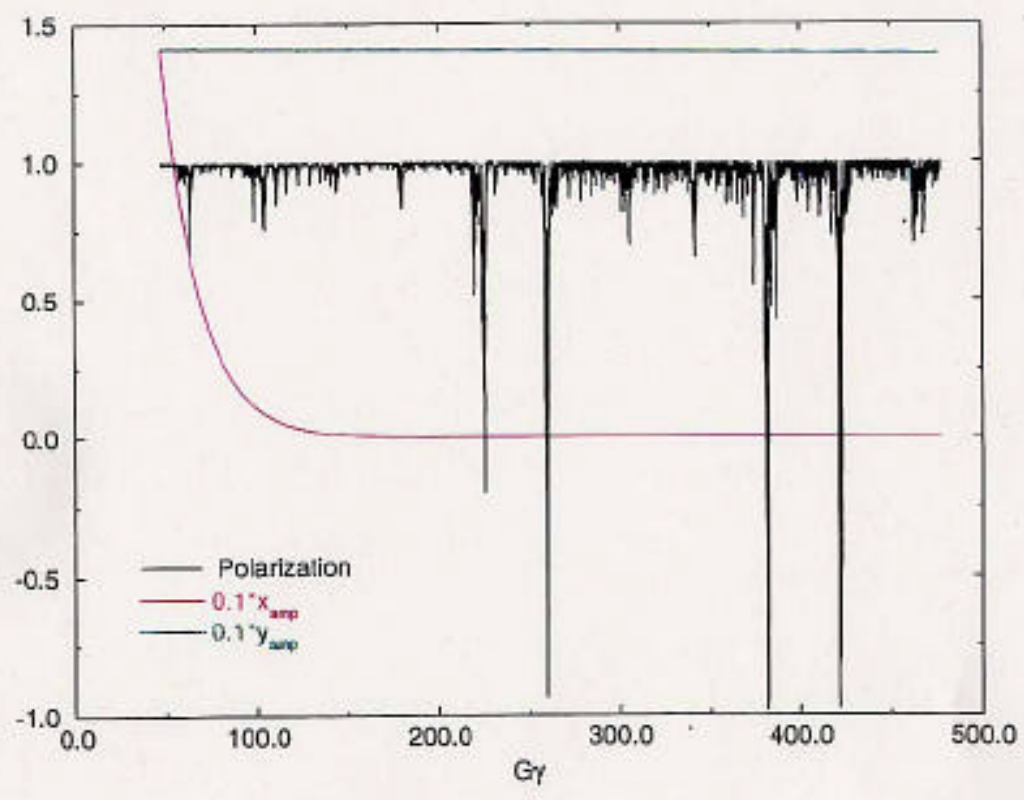

b. $\varepsilon_{\mathrm{x}}=\varepsilon_{\mathrm{y}}=20 \pi \mathrm{mm} \cdot \mathrm{mrad}$

Fig 2 Results of spin tracking with perfect Snakes in RHIC. Orbital tracking has been done with the "transport" matrices directly from Code MAD without symplectification. Normalized beam amplitudes are calculated at the $\beta^{*} \mathrm{~T}_{-} \mathrm{x}=9.956 \mathrm{~m}, \beta^{*} \mathrm{~T}_{-} \mathrm{y}=10.044 \mathrm{~m}$ 


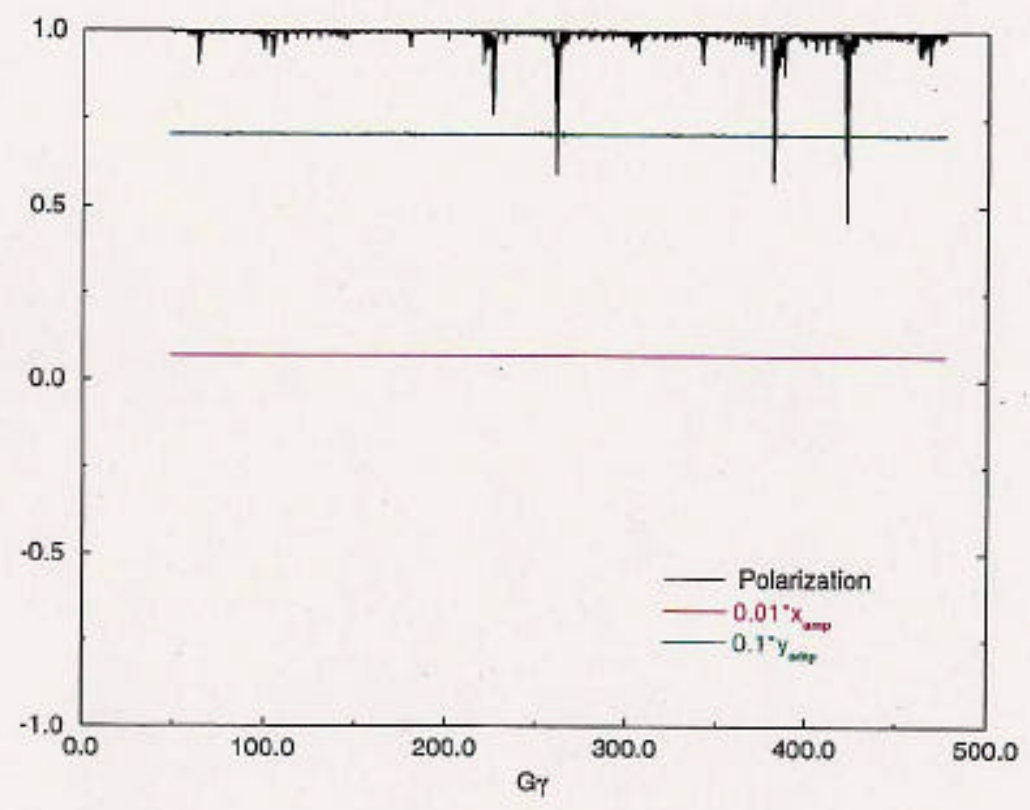

a. $\varepsilon_{x}=\varepsilon_{y}=5 \pi \mathrm{mm} \cdot \mathrm{mrad}$

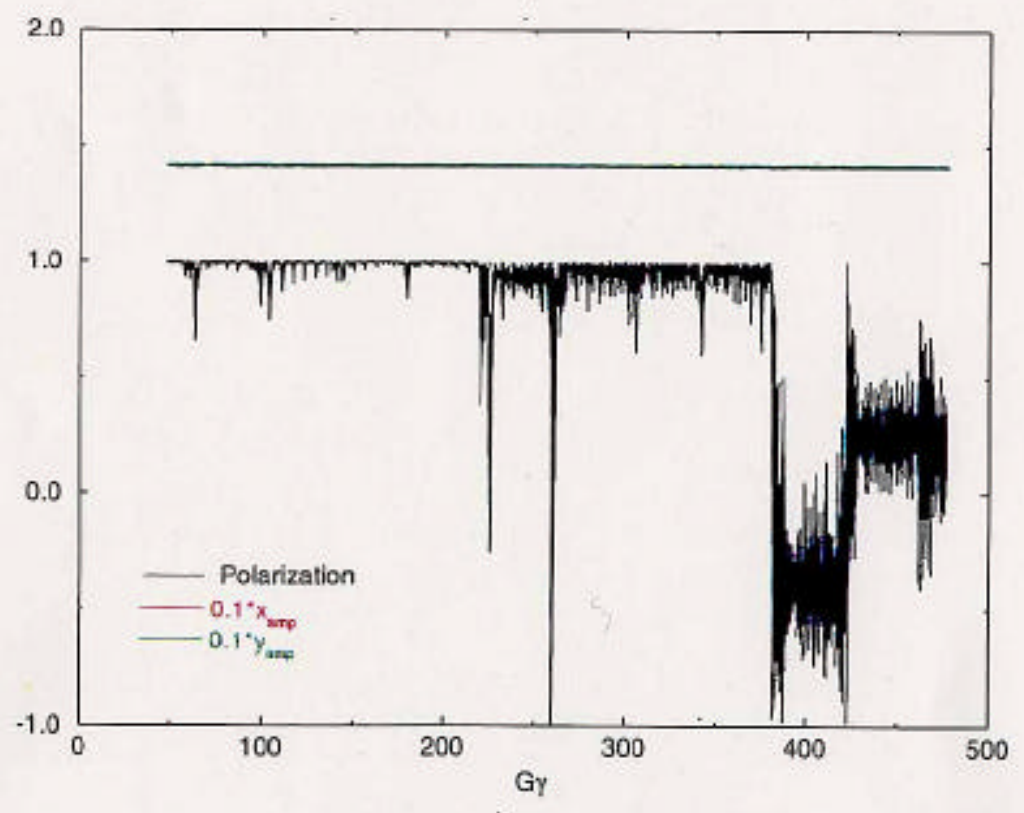

b. $\varepsilon_{\mathrm{x}}=\varepsilon_{\mathrm{y}}=20 \pi \mathrm{mm} \cdot \mathrm{mrad}$

Fig 3 Results of spin tracking with perfect Snakes in RHIC. Orbital tracking has been done with the "transport" matrices directly from Code MAD with symplectification. Normalized beam amplitudes are calculated at the $\beta^{*} \mathrm{~T}_{-} \mathrm{x}=9.956 \mathrm{~m}, \beta^{*} \mathrm{~T}_{-} \mathrm{y}=10.044 \mathrm{~m}$ 


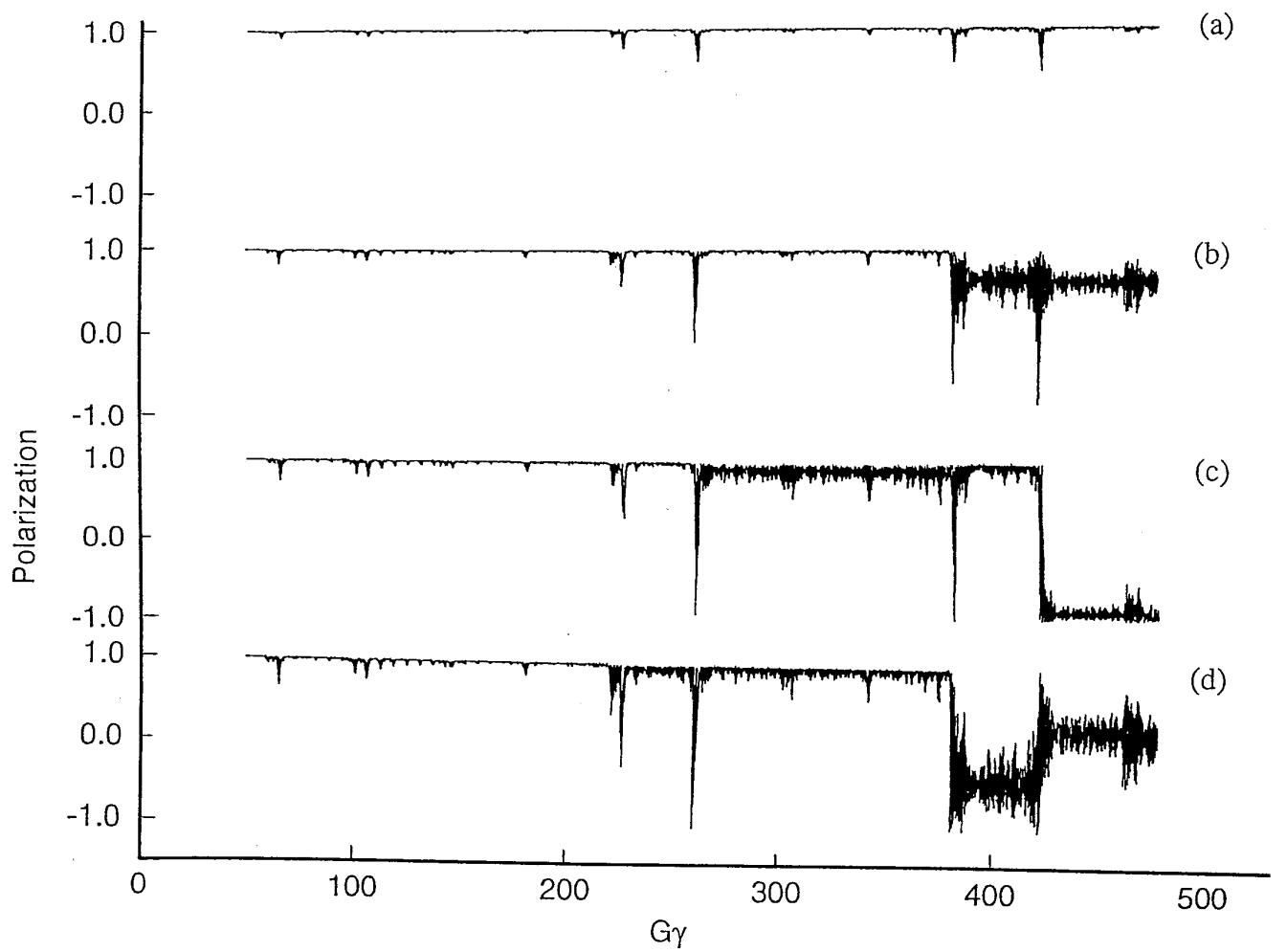

Fig 4 Spin tracking with perfect Snakes in RHIC, orbital tracking with the symplectified "transport" matrices from Code MAD

a. $\varepsilon_{\mathrm{x}}=\varepsilon_{\mathrm{y}}=5 \pi \mathrm{mm} \cdot \mathrm{mrad}, \quad b . \varepsilon_{\mathrm{x}}=\varepsilon_{\mathrm{y}}=10 \pi \mathrm{mm} \cdot \mathrm{mrad}$, c. $\varepsilon_{\mathrm{x}}=\varepsilon_{\mathrm{y}}=15 \pi \mathrm{mm} \cdot \mathrm{mrad}, \quad$ d. $\varepsilon_{\mathrm{x}}=\varepsilon_{\mathrm{y}}=20 \pi \mathrm{mm} \cdot \mathrm{mrad}$. 


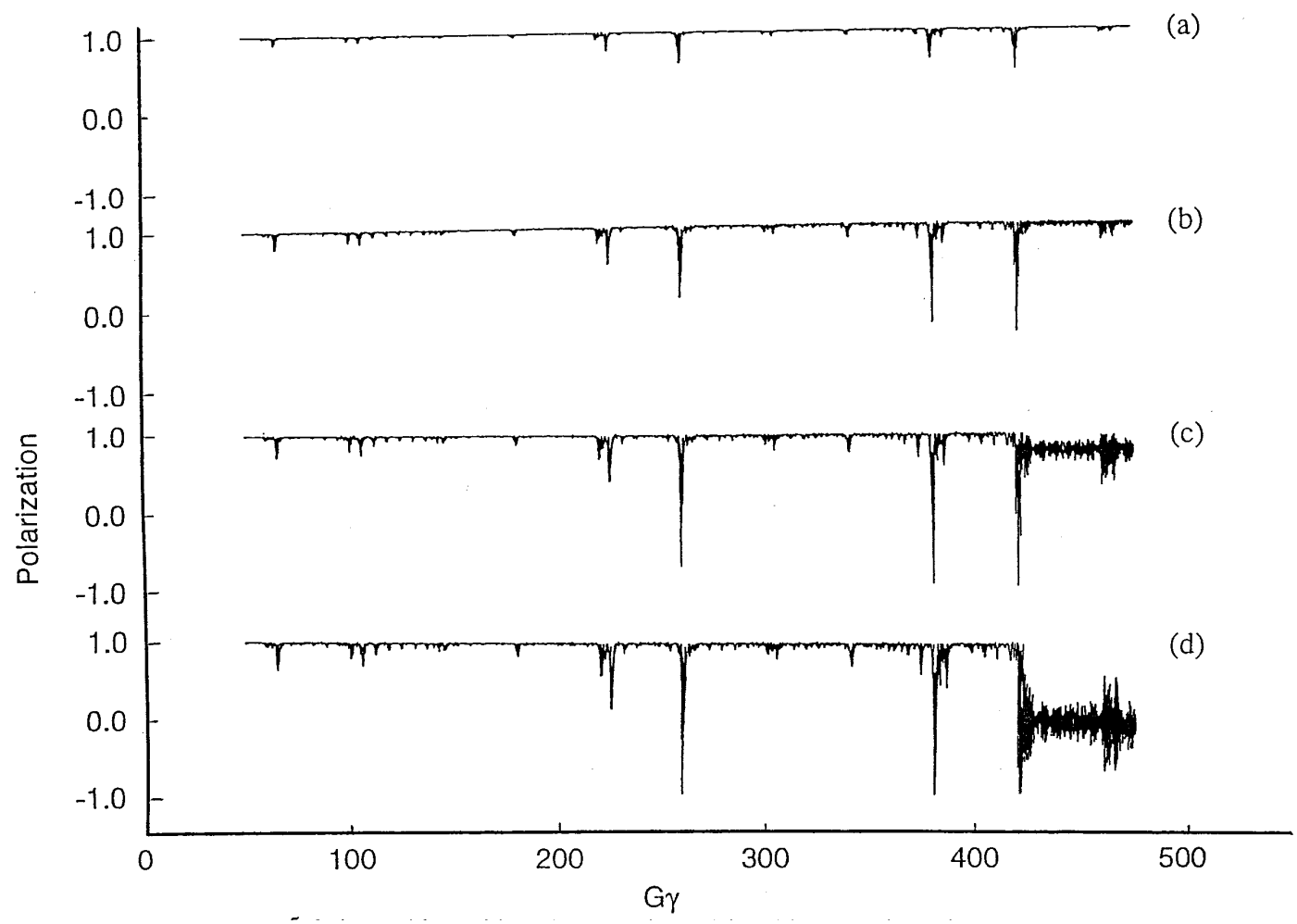

Fig 5 Spin tracking with real Snakes in RHIC, orbital tracking with the symplectified "transport" matrices from Code MAD

a. $\varepsilon_{\mathrm{x}}=\varepsilon_{\mathrm{y}}=5 \pi \mathrm{mm} \cdot \mathrm{mrad}, \quad b . \varepsilon_{\mathrm{x}}=\varepsilon_{\mathrm{y}}=10 \pi \mathrm{mm} \cdot \mathrm{mrad}$,

c. $\varepsilon_{\mathrm{x}}=\varepsilon_{\mathrm{y}}=15 \pi \mathrm{mm} \cdot \mathrm{mrad}, \quad$ d. $\varepsilon_{\mathrm{x}}=\varepsilon_{\mathrm{y}}=20 \pi \mathrm{mm} \cdot \mathrm{mrad}$. 


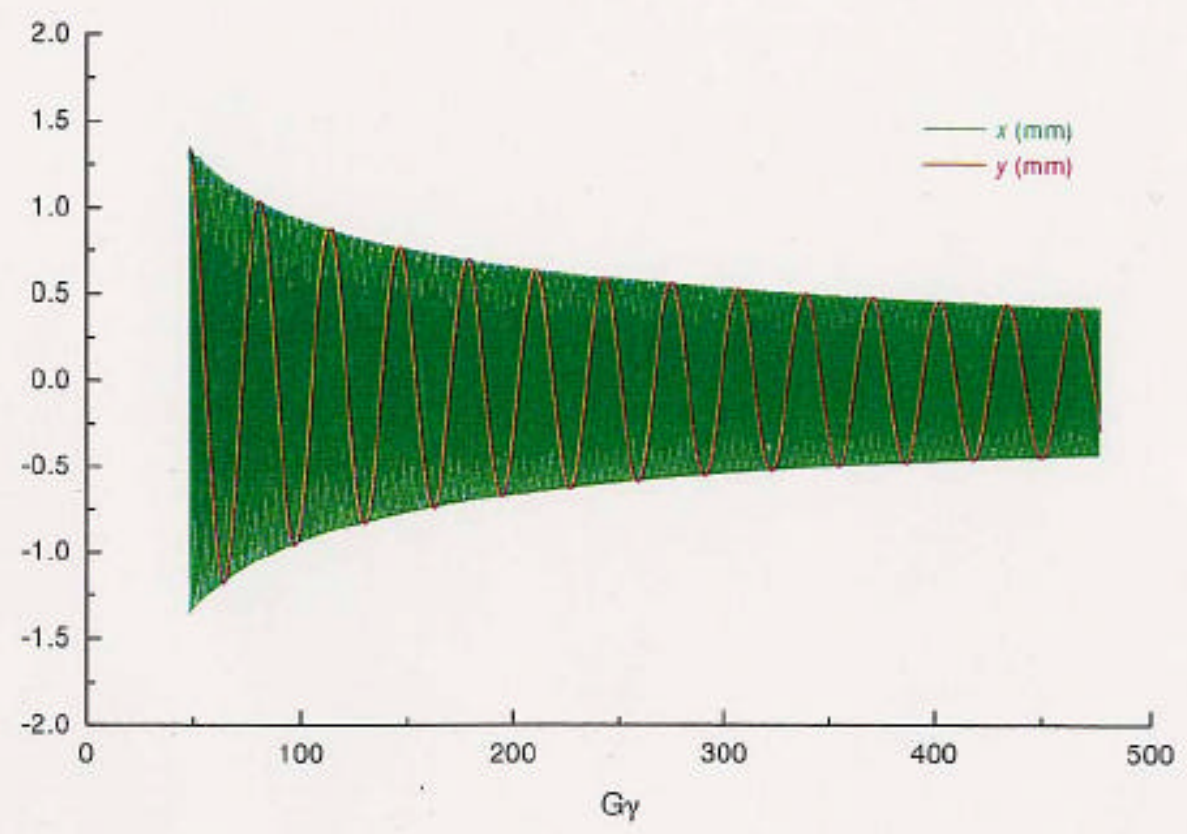

(a) with perfect Siberian Snakes

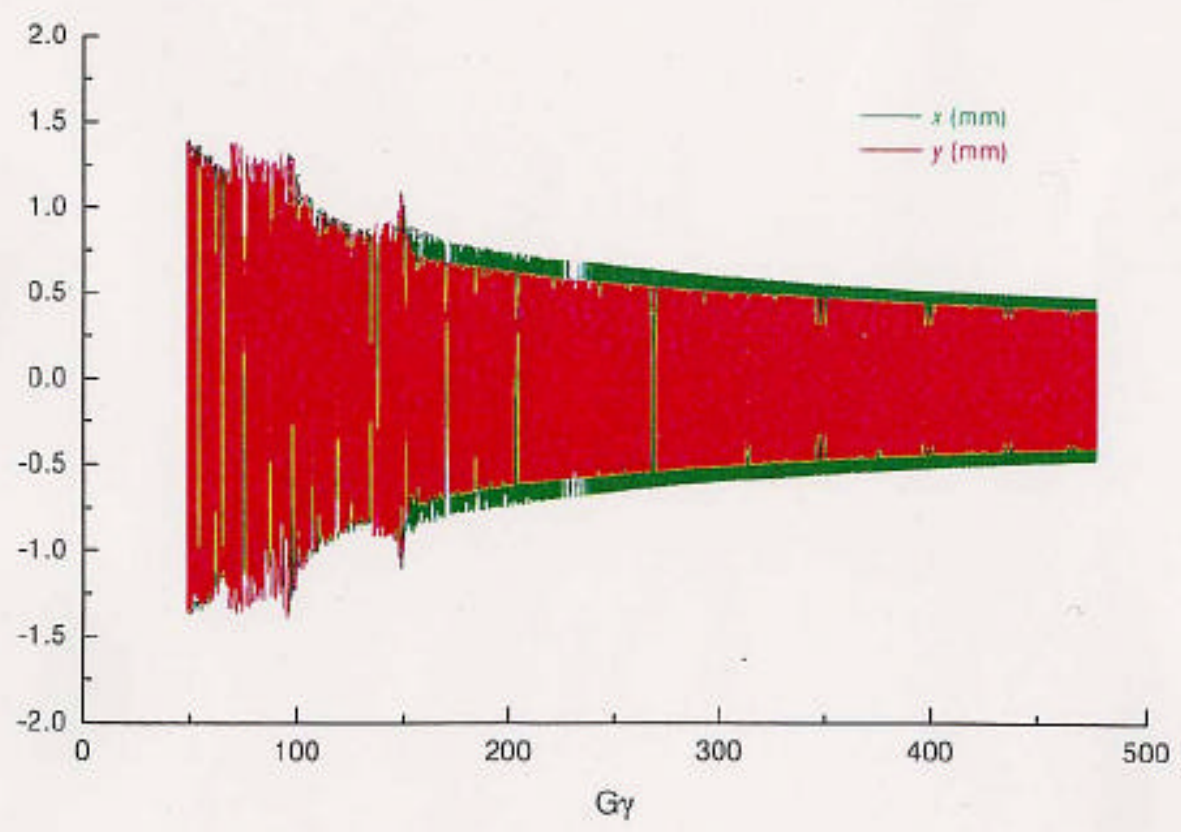

(b) with "real "Siberian Snakes

Fig 6 Betatron amplitude in $\mathrm{x}$ and $\mathrm{y}$ directions at 6'oclock in the RHIC ring Original emittance: $5 \pi \mathrm{mm} . \mathrm{mrad}$. 

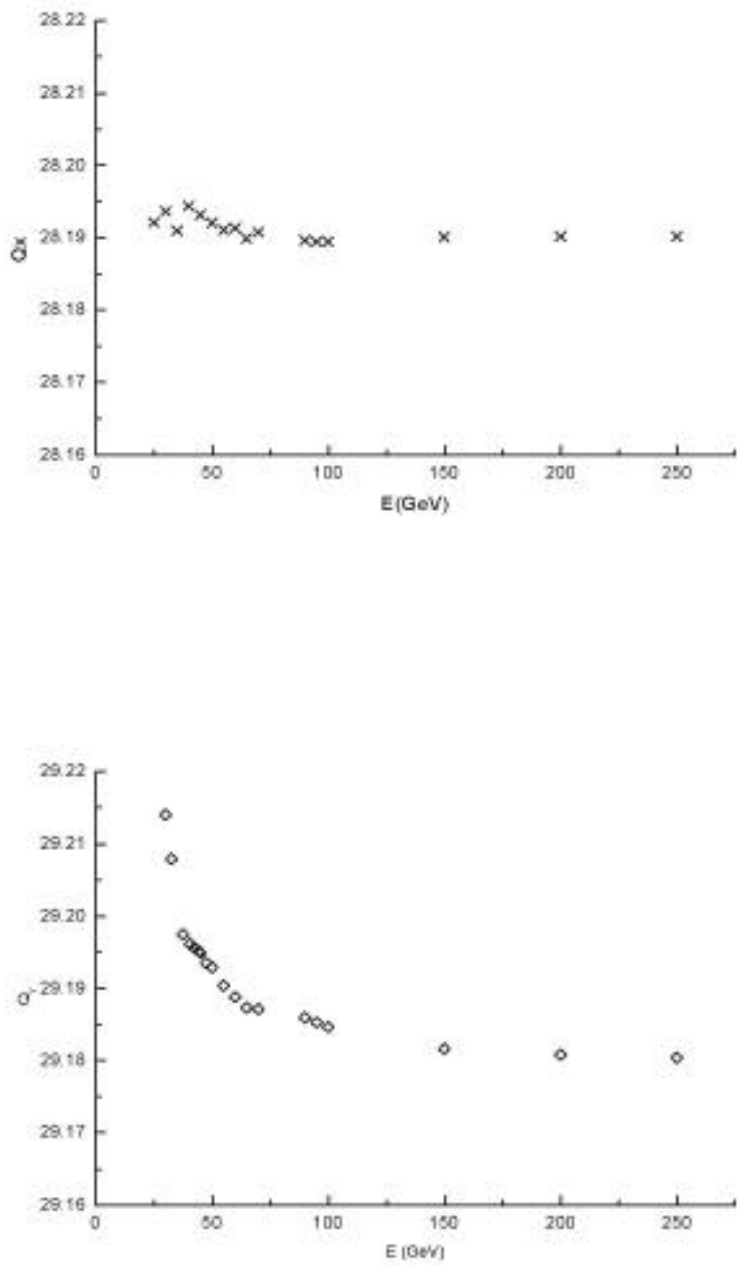

Fig 7 The transverse betatron tunes $Q_{x}$ and $Q_{\mathrm{y}}$ shifts through the acceleration 


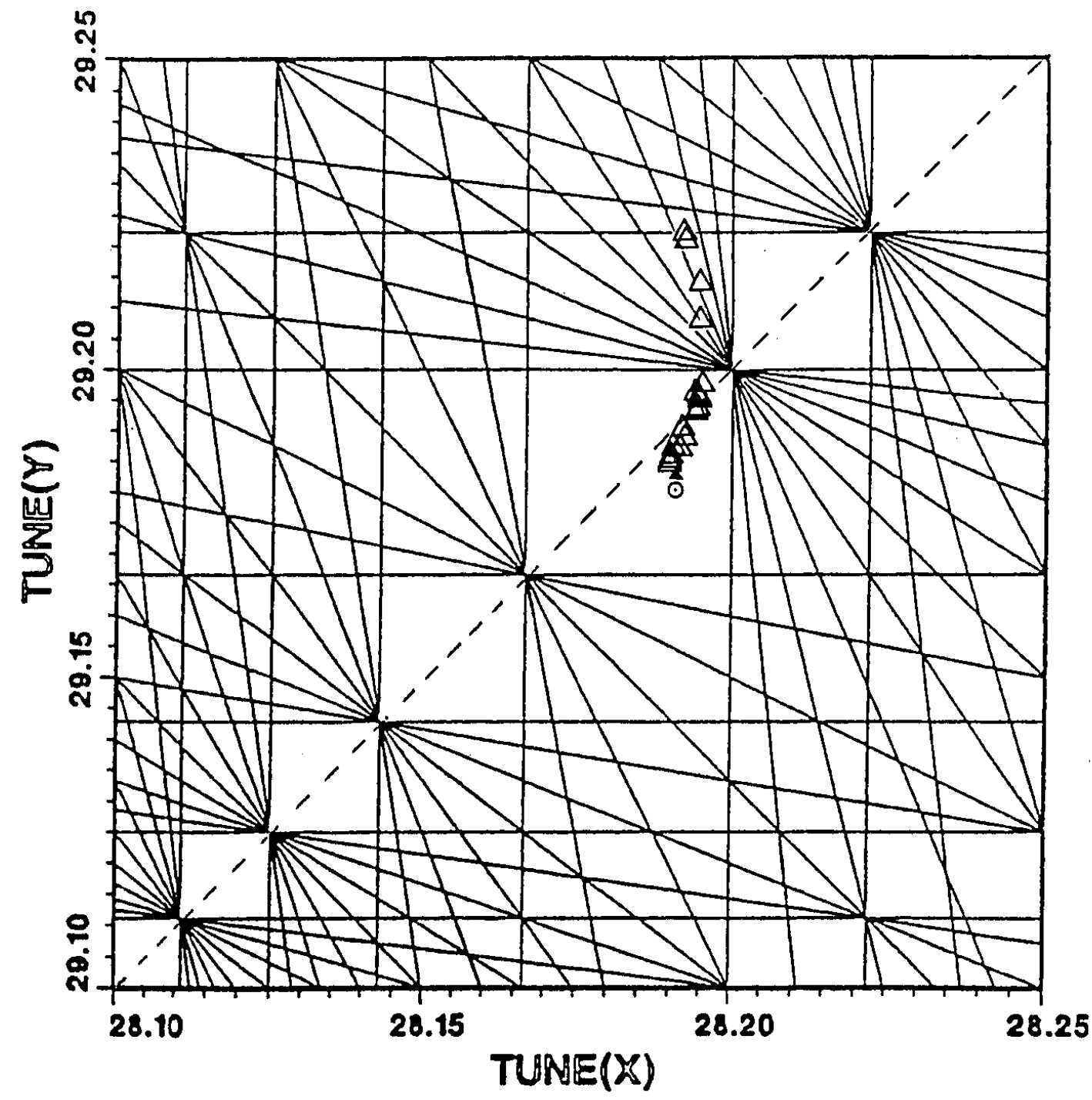

Fig 8 Tune diagram showing the selected working point of RHIC at $Q_{x}=28.19$ and $Q_{\mathrm{y}}=$ 29.18 (with symbol "o") with neighboring sum and difference resonances. The shifted working point due to the insertion of Snakes are shown with symbol " $\Delta$ " in the diagram. 

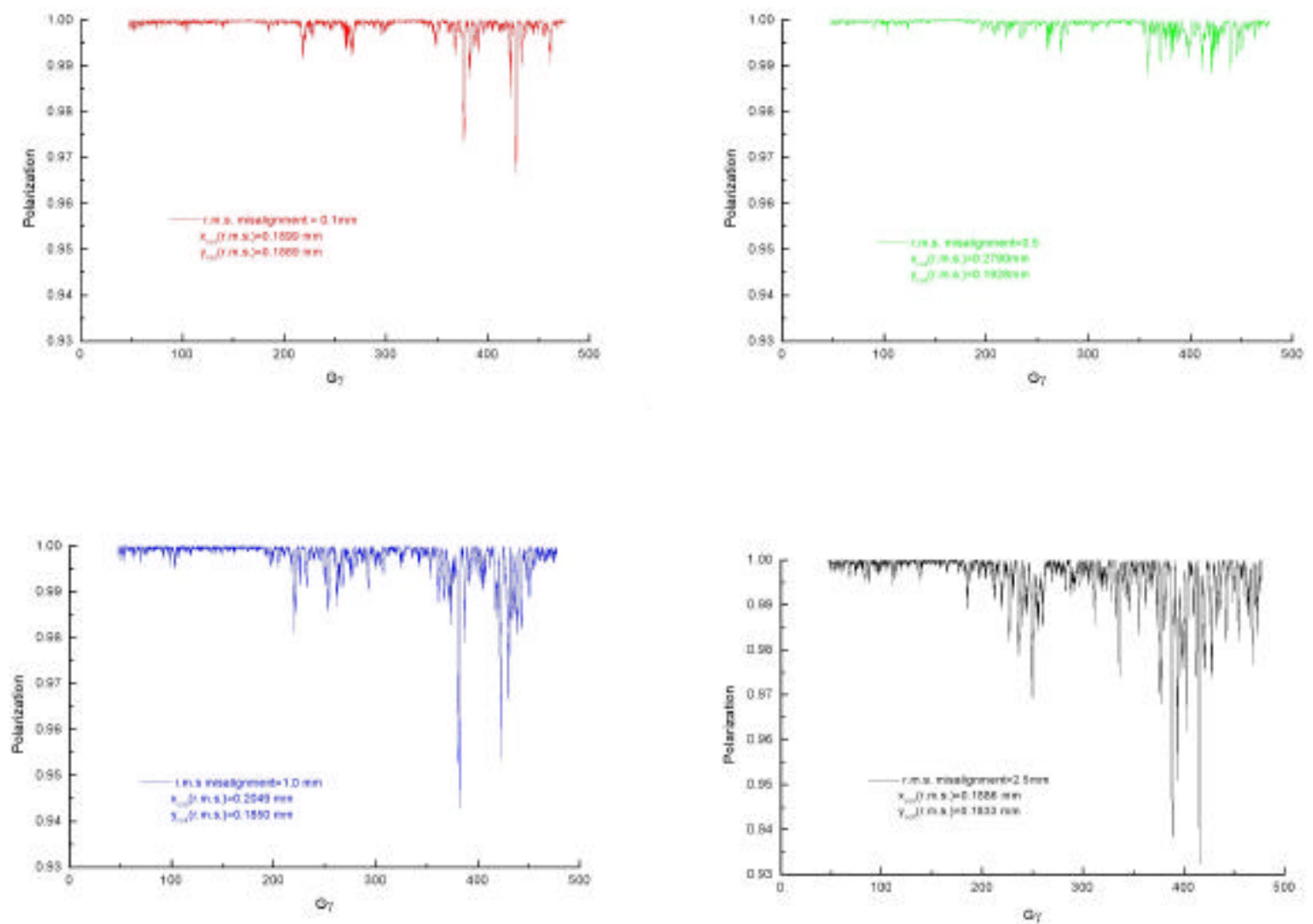

Fig 9 The effect of the misalignments of RHIC on spin motion. Spin tracking was done by single particle extracted from the emmittance of zero. Corrected vertical closed orbit distortions $\approx 0.18 \mathrm{~mm}$. Original r.m.s. misalignments are (a) $0.1 \mathrm{~mm}$, (b) $0.5 \mathrm{~mm}$, (c) $1.0 \mathrm{~mm}$, and (d) $2.5 \mathrm{~mm}$ 


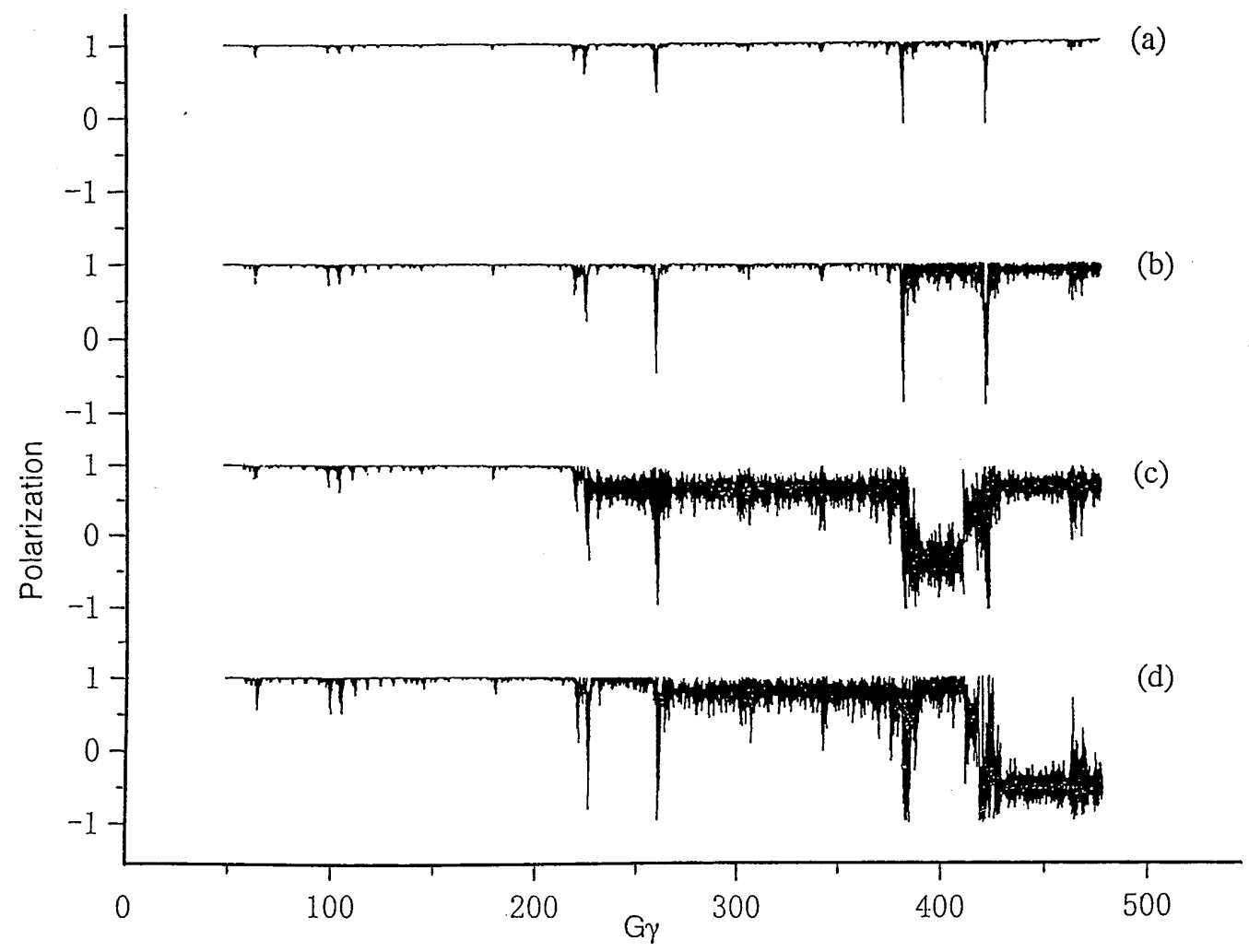

Fig 10 Spin tracking with real Snakes in RHIC, orbital tracking with the symplectified "transport" matrices from Code MAD

Misalignment randomly assigned,

Corrected Closed Orbit Distortion $\approx 0.2 \mathrm{~mm}$

$a$. $\varepsilon_{\mathrm{x}}=\varepsilon_{\mathrm{y}}=5 \pi \mathrm{mm} \cdot \mathrm{mrad}, \quad b . \varepsilon_{\mathrm{x}}=\varepsilon_{\mathrm{y}}=10 \pi \mathrm{mm} \cdot \mathrm{mrad}$,

c. $\varepsilon_{\mathrm{x}}=\varepsilon_{\mathrm{y}}=15 \pi \mathrm{mm} \cdot \mathrm{mrad}, \quad d . \varepsilon_{\mathrm{x}}=\varepsilon_{\mathrm{y}}=20 \pi \mathrm{mm} \cdot \mathrm{mrad}$. 


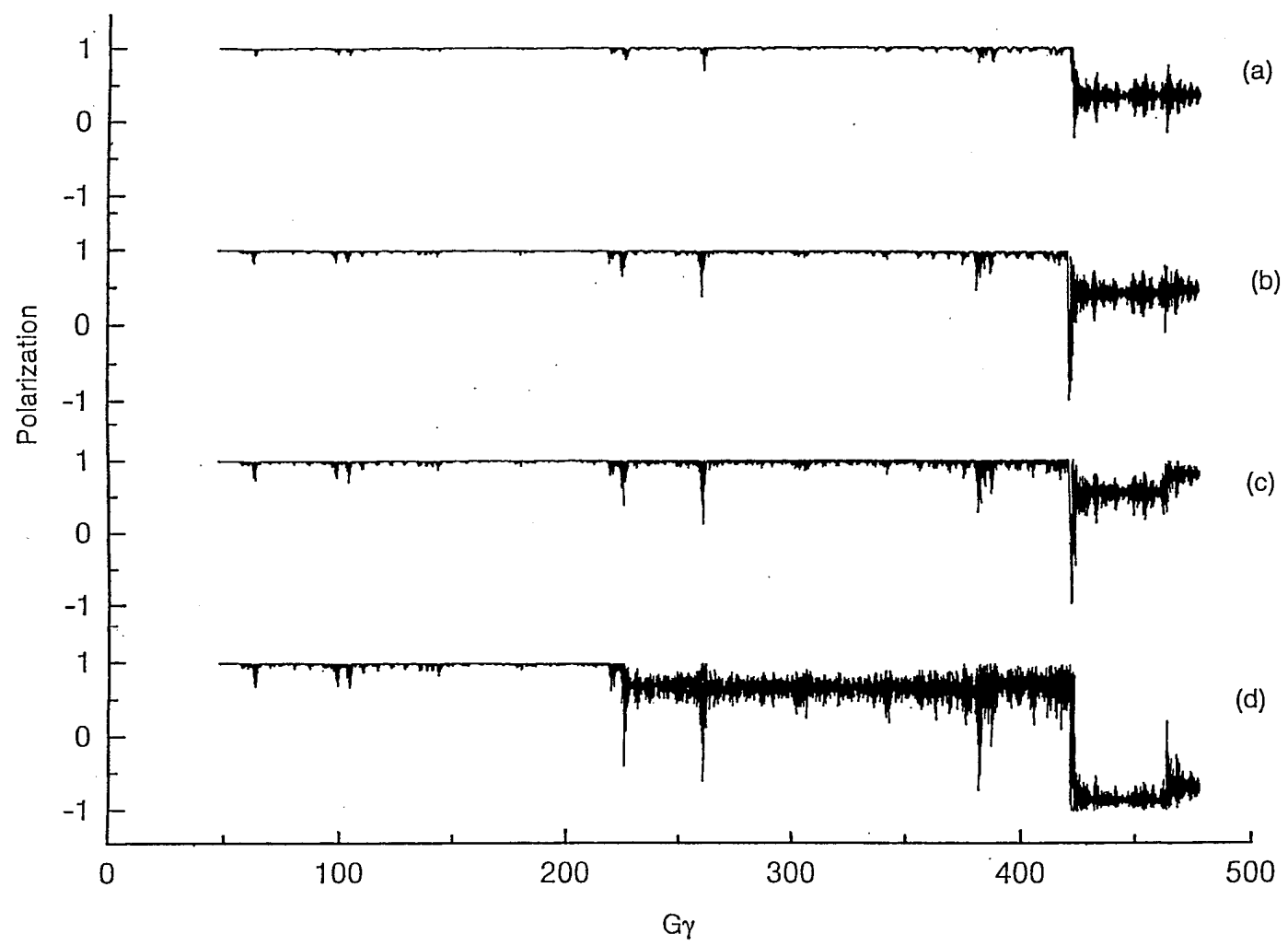

Fig 11 Spin tracking with real Snakes in RHIC, orbital tracking with the symplectified "transport" matrices from Code MAD Misalignment randomly assigned,

Corrected Closed Orbit Distortion $\approx 0.5 \mathrm{~mm}$
a. $\varepsilon_{\mathrm{x}}=\varepsilon_{\mathrm{y}}=5 \pi \mathrm{mm} \cdot \mathrm{mrad}$
b. $\varepsilon_{\mathrm{x}}=\varepsilon_{\mathrm{y}}=10 \pi \mathrm{mm} \cdot \mathrm{mrad}$,
c. $\varepsilon_{\mathrm{x}}=\varepsilon_{\mathrm{y}}=15 \pi \mathrm{mm} \cdot \mathrm{mrad}$,
d. $\varepsilon_{\mathrm{x}}=\varepsilon_{\mathrm{y}}=20 \pi \mathrm{mm} \cdot \mathrm{mrad}$ 


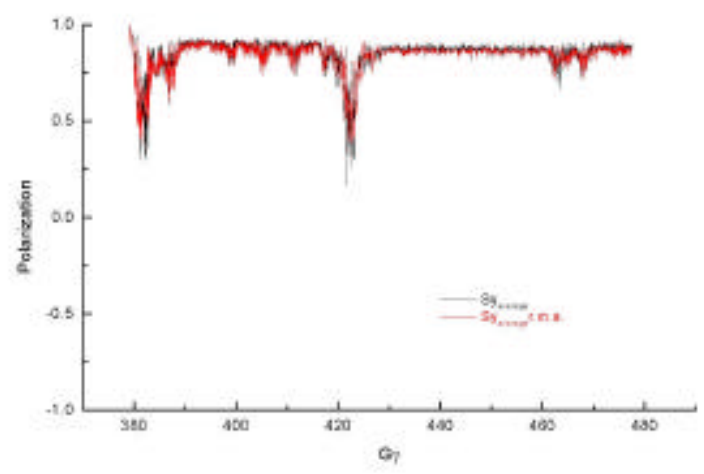

(a) with "perfect" snakes in RHIC

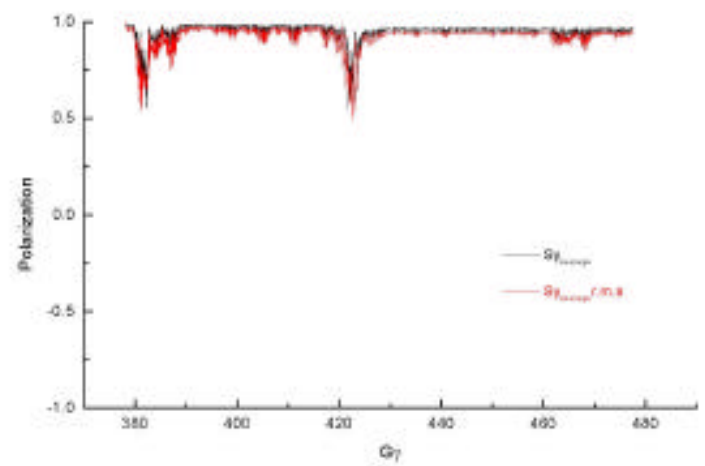

(b) with "perfect" snakes in RHIC

Fig 12 The average results of polarization from 32 particles's tracking in RHIC with No misalignment and no field errors. Original emittance: $10 \pi \mathrm{mm} . \mathrm{mrad}$.

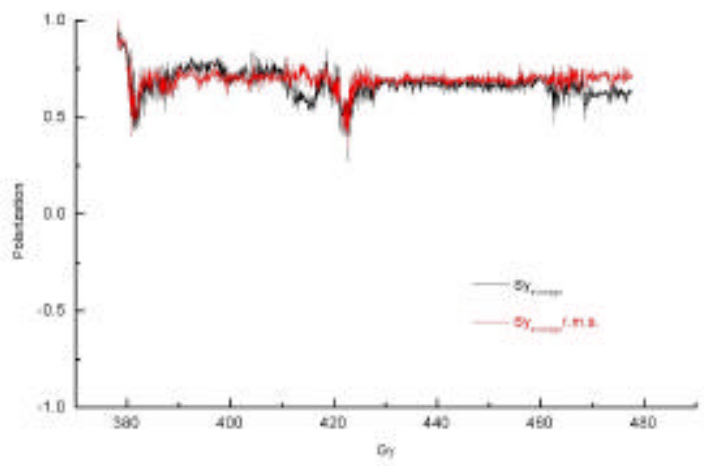

Fig 13 The average results of polarization from 32 particles's tracking in RHIC with r.m.s. misalignment of $2.5 \mathrm{~mm}$ and corrected vertical closed orbit distortion of $0.189 \mathrm{~mm}$. Original emittance: $10 \pi \mathrm{mm} . \mathrm{mrad}$. 